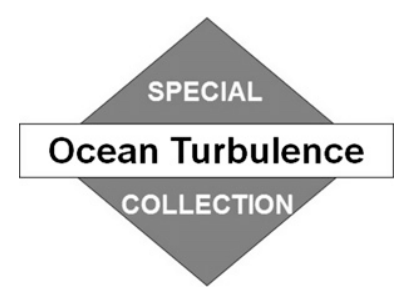

\title{
Sparse Sampling of Intermittent Turbulence Generated by Breaking Surface Waves
}

\author{
MORTEZA DERAKHTI AND JIM THOMSON \\ Applied Physics Laboratory, University of Washington, Seattle, Washington \\ JAMES T. KIRBY \\ Center for Applied Coastal Research, University of Delaware, Newark, Delaware
}

(Manuscript received 10 June 2019, in final form 10 January 2020)

\begin{abstract}
We examine how Eulerian statistics of wave breaking and associated turbulence dissipation rates in a field of intermittent events compare with those obtained from sparse Lagrangian sampling by surface following drifters. We use a polydisperse two-fluid model with large-eddy simulation (LES) resolution and volume-offluid surface reconstruction (VOF) to simulate the generation and evolution of turbulence and bubbles beneath short-crested wave breaking events in deep water. Bubble contributions to dissipation and momentum transfer between the water and air phases are considered. Eulerian statistics are obtained from the numerical results, which are available on a fixed grid. Next, we sample the LES/VOF model results with a large number of virtual surface-following drifters that are initially distributed in the numerical domain, regularly or irregularly, before each breaking event. Time-averaged Lagrangian statistics are obtained using the time series sampled by the virtual drifters. We show that convergence of statistics occurs for signals that have minimum length of approximately 1000-3000 wave periods with randomly spaced observations in time and space relative to three-dimensional breaking events. We further show important effects of (i) extent of measurements over depth and (ii) obscuration of velocity measurements due to entrained bubbles, which are the two typical challenges in most of the available in situ observations of upper ocean wave breaking turbulence. An empirical correction factor is developed and applied to the previous observations of Thomson et al. Applying the new correction factor to the observations noticeably improves the inferred energy balance of wind input rates and turbulence dissipation rates. Finally, both our simulation results and the corrected observations suggested that the total wave breaking dissipation rates have a nearly linear relation with active whitecap coverage.
\end{abstract}

\section{Introduction}

Many previous studies have shown that turbulence dissipation rates in the ocean surface layer are elevated in the presence of breaking waves (e.g., Agrawal et al. 1992; Terray et al. 1996; Gemmrich and Farmer 2004; Gemmrich 2010; Thomson 2012; Sutherland and Melville 2015). This turbulence is important as an input of energy from the wind to the ocean (Gemmrich et al. 1994), a sink of energy for the surface waves (Melville 1996), and a driver of air-sea gas exchange (Zappa et al. 2007). The turbulence is complicated by two-phase flow, in which bubbles are active particles (e.g., Rapp and Melville 1990; Lamarre and Melville 1991; Derakhti and Kirby 2014a;

\footnotetext{
Corresponding author: Morteza Derakhti, derakhti@uw.edu
}

Deike et al. 2017a). Another challenge is the intermittent nature of the forcing, with individual waves breaking as a result of random phase interference patterns and modulational instability (Babanin 2011).

Direct measurements of the turbulence beneath breaking surface waves are rare. Recent examples have employed a surface-following reference frame (e.g., Gemmrich 2010; Thomson 2012; Sutherland and Melville 2015; Zippel et al. 2018), which is a natural choice for the observations but a challenge to reconcile with the fixed (Eulerian) reference frames common in numerical models. Furthermore, the observations generally are sparse in space and time, such that it has been difficult to ensure robust statistics. Published observations of turbulence in the ocean near-surface layer generally find that 1) turbulence levels greatly exceed those predicted 
by law-of-the-wall shear scaling, and 2) this waveenhanced layer is limited to a depth of approximately one significant wave height in a fixed reference frame (or 1-2 $\mathrm{m}$ in a wave-following reference frame) (Esters et al. 2018). As most of these observations use acoustic Doppler methods to obtain turbulent fluid velocities, the data in the most active portion of the breaking waves are often occluded by bubbles. Thus, existing observations likely represent an incomplete average of the surface conditions, which lack the maxima occurring in space and time.

Numerical models and laboratory experiments have been essential in filling the gaps, for example, quantifying turbulence-bubble interaction in bubbly flows beneath breaking waves and providing a high-resolution spatiotemporal variation of turbulence dissipation rates during active breaking. The early studies of Rapp and Melville (1990) and Lamarre and Melville (1991) established time and length scales for the turbulence from two-dimensional focused wave packets, including the importance of bubbles in the setting of the total dissipation. More recently, Wang and Wijesekera (2018) conducted a large-scale laboratory experiment with three-dimensional (3D), that is, short-crested, breaking crests in a modulated wave train. Their measurements showed that values of near-surface turbulence dissipation rates during an active breaking event is from two to three orders of magnitude larger than those before and after the wave breaking. The recent numerical efforts of Derakhti and Kirby (2014a, 2016) and Deike et al. (2016, 2017a) resolve the breaking of individual waves and the associated turbulence and bubble dynamics. Derakhti and Kirby (2014a) results showed that high dissipation rates occur preferentially in regions with high void fraction within bubble plumes. Furthermore, their simulation results predicted that bubble-induced dissipation accounts for approximately $50 \%$ of the total wavebreaking-induced turbulence dissipation regardless of breaker type and intensity. Here, bubble-induced dissipation refers to the enhancement of turbulence dissipation due to the subgrid-scale (SGS) turbulent motions generated by the dispersed bubbles (see Derakhti and Kirby 2014a, section 4.3.1 for more details).

The present work is motivated by the study of Thomson et al. (2016), in which turbulence dissipation rates were estimated using Doppler velocity profiles within the upper meter of the wave-following surface. That study concluded that strong turbulence is isolated to a very thin layer $(<1 \mathrm{~m})$, but that orbital motions advect the turbulence over vertical scales of at least one significant wave height. The main focus of Thomson et al. (2016) was evaluating the energetic balance at the surface, with the conclusion that the observed energy dissipation rates were insufficient to balance the energy input rates using several different formulations.

Here, we revisit the topics of Thomson et al. (2016) by sampling a high-fidelity numerical model in the Lagrangian mode of the surface-following observations. We use a polydisperse two-fluid model (Derakhti and Kirby 2014a) with large-eddy simulation (LES) resolution and volume-of-fluid surface reconstruction (VOF) to simulate the generation and evolution of turbulence and bubbles beneath $3 \mathrm{D}$ short-crested wave breaking events in deep water (section 2). We first scale the model domain to match the observed whitecap coverage values, and we scale the model wave heights to match the wind-wave (i.e., equilibrium) portion of the observed spectrum (i.e., neglecting swell). We then determine the effects of sparse sampling and intermittent breaking, as well as the effects of data occlusion by bubbles and limitations in the vertical extent of the observed profiles (section 3). In section 4, we comment on the apparent discrepancy between the observed windinput energy fluxes and total turbulence dissipation rates reported by Thomson et al. (2016). Examination of potential Lagrangian sampling bias related to a partially trapped drifter in convergence zones in the turbulence observations is left for future work.

\section{Methods}

In this section, we first present the model governing equations for continuity of mass and momentum of liquid and gas phases of a polydisperse two-fluid mixture, as described in Derakhti and Kirby (2014a). The model setup including details of the incident wave conditions and the scaling of the model domain to match observations of whitecap coverage are then described. Finally, we explain our methodology to convert the model results to surface following virtual drifters.

Demonstrations of model convergence and performance, including detailed comparisons of free surface evolution, bubble void fraction, integral properties of the bubble plume, organized and turbulent velocity fields and total wave-breaking-induced energy dissipation, for various deep- and shallow-breaking waves may be found in Derakhti and Kirby (2014a,b, 2016) and Derakhti et al. (2018, 2019, manuscript submitted to J. Geophys. Res. Oceans).

\section{a. Mathematical formulations}

The computations here are performed using the LES/VOF Navier-Stokes solver Truchas (Francois et al. 2006) with extensions of a polydisperse bubble phase and various turbulence closures (Carrica et al. 1999; Ma et al. 2011; Derakhti and Kirby 2014a). Details of the 
mathematical formulations and numerical method may be found in Derakhti and Kirby (2014a, section 2).

The filtered governing equations for continuity of mass and momentum of the liquid phase are given by (Derakhti and Kirby 2014a)

$$
\begin{gathered}
\frac{\partial \alpha \rho}{\partial t}+\frac{\partial \alpha \rho \tilde{u}_{j}}{\partial x_{j}}=0, \\
\frac{\partial \alpha \rho \tilde{u}_{i}}{\partial t}+\frac{\partial \alpha \rho \tilde{u}_{i} \tilde{u}_{j}}{\partial x_{j}}=\frac{\partial \Pi_{i j}}{\partial x_{j}}+\alpha \rho g \delta_{3 i}+\mathbf{M}^{\mathrm{gl}},
\end{gathered}
$$

where $(i, j)=1,2,3 ; \rho$ is a constant liquid density; $\alpha$ and $\tilde{u}_{i}$ are the volume fraction and the filtered velocity in the $i$ direction of the liquid phase respectively; $\delta_{i j}$ is the Kronecker delta function; $g$ is the gravitational acceleration; and $\Pi_{i j}=\alpha\left(-\tilde{p} \delta_{i j}+\tilde{\sigma}_{i j}-\rho \tau_{i j}\right)$ with $\tilde{p}$ the filtered pressure, which is identical in each phase due to the neglect of interfacial surface tension, $\tilde{\sigma}_{i j}$ viscous stress, and $\tau_{i j}$ the SGS stress estimated using an eddy viscosity assumption and the dynamic Smagorinsky model, which includes liquid/bubble interaction effects (for more details see Derakhti and Kirby 2014a, section 2.4). Finally, $\mathbf{M}^{\mathrm{gl}}$ are the momentum transfers between liquid and gas phases, including the filtered virtual mass, lift, and drag forces (Derakhti and Kirby 2014a).

Using the same filtering process as in the liquid phase, the equations for the bubble number density and continuity of momentum for each bubble size class with a diameter $d_{k}^{b}, k=1, \ldots, N_{G}$, are then given by (Derakhti and Kirby 2014a)

$$
\begin{aligned}
\frac{\partial N_{k}^{b}}{\partial t}+\frac{\partial \tilde{u}_{k, j}^{b} N_{k}^{b}}{\partial x_{j}} & =R_{k}^{b}, \\
0 & =-\frac{\partial \alpha_{k}^{b} \tilde{p}}{\partial x_{j}} \delta_{i j}+\alpha_{k}^{b} \rho^{b} g_{i}+\mathbf{M}_{k}^{\mathrm{gg}},
\end{aligned}
$$

where $m_{k}^{b}, N_{k}^{b}, \alpha_{k}^{b}=m_{k}^{b} N_{k}^{b} / \rho^{b}$, and $\tilde{u}_{k, j}^{b}$ are the mass, number density, volume fraction, and filtered velocity in the $j$ direction of the $k$ th bubble size class; $\rho^{b}$ is the bubble density; and $R_{k}^{b}$ includes the source due to air entrainment in the interfacial cells (Derakhti and Kirby 2014a, section 2.3), intergroup mass transfer, and SGS diffusion terms. Finally, $\mathbf{M}_{k}^{\mathrm{lg}}$ represents the total momentum transfer between liquid and the $k$ th bubble size class, and satisfies $\mathbf{M}^{\mathrm{gl}}+\sum_{k=1}^{N_{G}} \mathbf{M}_{k}^{\mathrm{lg}}=0$. In Eq. (4), we neglect the inertia and shear stress terms in the gas phase following Carrica et al. (1999) and Derakhti and Kirby (2014a).

\section{b. Model setup}

Our numerical experiments are carried out in a virtual wave tank of unperturbed constant depth $h$, extending a length $L_{x}$ in the $x$ direction, and $\pm L_{y} / 2$ in the transverse $y$ direction. The vertical direction $z$ in the fixed reference frame is positive upward and measured from the still water level. The virtual wave tank is sufficiently deep to avoid any depth-limited wave breaking, such that the experiments remain focused on whitecaps.

All simulations are performed with the model initialized with quiescent conditions. An incident wave packet is then generated at the model upstream boundary $(x=0)$. The input focused wave packet was composed of $N=10$ sinusoidal components of steepness $a_{n} k_{n}$, $n=1, \ldots, N$, where $a_{n}$ and $k_{n}$ are the amplitude and wavenumber of the $n$th frequency component. The steepness of individual wave components is taken to be constant across the spectrum, or $a_{1} k_{1}=a_{i} k_{i}=\ldots=$ $a_{N} k_{N}=S_{g} / N$ with $S_{g}=\sum_{n=1}^{N} a_{n} k_{n}$ taken to be a measure of the wave train global steepness. Based on linear theory, the free surface elevation for the 3D shortcrested focused packets (Wu and Nepf 2002; Derakhti et al. 2018; Kirby and Derakhti 2019) at the wavemaker is given by

$$
\eta(0, y, t)=\sum_{n=1}^{N} a_{n} \cos \left[2 \pi f_{n}\left(t-t_{f}\right)+\frac{k_{n} x_{f}}{\cos \theta(y)}\right],
$$

where $f_{n}$ is the frequency of the $n$th component, $x_{f}$ and $t_{f}$ are the predefined, linear theory estimates of location and time of the focal point respectively, and $\theta(y)$ is the angle of incidence of each wave component at various transverse locations with $\cos \theta(y)=x_{f} / \sqrt{x_{f}^{2}+y^{2}}$. The discrete frequencies $f_{n}$ were uniformly spaced over the band $\Delta f=f_{N}-f_{1}$ with a central frequency defined by $f_{c}=\left(f_{N}+f_{1}\right) / 2$. Increasing the global steepness $S_{g}$ and/or decreasing $\Delta f l f_{c}$ increases the total wave energy loss due to the resulting breaking event(s) in the virtual tank. Finally, liquid velocities for each wave component are calculated using linear theory and then superimposed at the wavemaker.

We define the spectrally weighted frequency of the wave field $f_{s}$ as

$$
f_{s}=\frac{\int f E(f) d f}{\int E(f) d f},
$$

where $E\left(\mathrm{~m}^{2} \mathrm{~s}\right)$ is the power spectral density of the wave field. The characteristic wavelength $L_{s}$ and period $T_{s}$ are then calculated based on $f_{s}$ and using the linear dispersion relation (as in Tian et al. 2010; Derakhti and Kirby 2016). The reference $x$ location $x^{*}$ is taken as the location at which the first forward-moving jet of considered breaking events in a numerical case hits the undisturbed 
TABLE 1. Input parameters for the simulated short-crested (3D) focused wave packets. In all three cases $N=10, \Delta f / f_{c}=0.75, T_{g} / T_{s}$ $\approx 9.5, x_{f} / L_{s} \approx 1.6-1.9, y_{f}=0$, and $t_{f}=15.0 \mathrm{~s}$. Definitions of all parameters presented here are given in section $2 \mathrm{~b}$.

\begin{tabular}{cccccccc}
\hline \hline Case No. & $S_{g}$ & $f_{c}\left(\mathrm{~s}^{-1}\right)$ & $T_{s}(\mathrm{~s})$ & $L_{s}(\mathrm{~m})$ & $h / L_{s}$ & $L_{x} / L_{s}$ & $L_{y} / 2 L_{s}$ \\
\hline T1 & 0.44 & 0.7 & 1.8 & 5.1 & 0.59 & 5 & 0.8 \\
T2 & 0.32 & 0.7 & 1.8 & 5.1 & 0.59 & 5 & 0.8 \\
T3 & 0.40 & 0.9 & 1.4 & 3.0 & 0.42 & 4 & 1.0 \\
\hline
\end{tabular}

free surface, and is normalized by $L_{s}$. Further, we define $y^{*}=y / L_{s}$ and $z^{*}=z / L_{s}$.

Each numerical case is defined by setting the geometry of the virtual tank and the input wave packet. Here, three representative cases are considered, with all relevant parameters summarized in Table 1 . In all cases, most of the wave components in the input packets are characterized as deep water waves.

Figure 1a shows the temporal variation of the normalized free surface elevations at the center of the tank and slightly upstream of the break point, $\left(x^{*}, y^{*}\right)=$ $(-0.1,0)$, for the case T1. The results indicate that the current wavemaker setup [Eq. (5)] results in a repeatable sequence of waves in the incident packet with a period of $T_{g}=N / \Delta f$. In all cases, the observed main breaking events in the virtual tank occur approximately every $T_{g}$. As shown in Figs. 1a, 1d, and 1e, however, the incident waves and the $x$ location of the main breaking event within each $T_{g}$ are not the same; this may be partially because of seiching in the virtual tank and reflections from the numerical boundaries.

We only consider the model results for $t>t_{0}$ for all the analyses presented in this paper, where $t_{0}>12 T_{g}>200 \mathrm{~s}$ is a time after which the background turbulence levels reach a quasi-steady state. For each case, we define the main breaking event $E_{m}\left(m=1,2, \ldots, N_{E}\right)$ as the most energetic breaking event that occurs in $m-1<(t-$ $\left.t_{0}\right) / T_{g}<m$. The total number of considered main breaking events $N_{E}$ for the cases T1, T2, and T3 are 9, 10, and 6 , respectively. Thus, considering an output sampling rate of $f_{\text {out }}$, the time series of all Eulerian variables predicted by the model have $N_{E} T_{g} f_{\text {out }}$ data points, where $f_{\text {out }}$ was $20 \mathrm{~Hz}$ for $\mathrm{T} 1$ and $\mathrm{T} 2$, and $25 \mathrm{~Hz}$ for $\mathrm{T} 3$.

\section{c. Matching the model and observed conditions}

We need to choose a number of well-defined parameters to present both the wave breaking forcing and model results in a nondimensional form, such that they can be appropriately scaled to field conditions. Here our goal is to have the wave spectrum $E$ and the fractional area of breaking crests of the simulated cases as consistent as possible with those observed in the field. The latter is usually referred to as the active part of the whitecap coverage $W$ of visible breaking crests, hereafter referred to as $W_{A}$, which is a space- and time-averaged quantity calculated over a given domain. There is a growing body of literature documenting a direct relationship between $W_{A}$ and the total wave breaking energy dissipation in the upper ocean (Callaghan et al. 2016, 2017; Callaghan 2018; Anguelova and Hwang 2016). We also need a characteristic breaking wave height to scale the vertical profiles of wave-breaking-related dynamical measures, such as the turbulence dissipation rates.

Figure $1 \mathrm{~b}$ shows the normalized power spectral density $E^{*}=E H_{\mathrm{eq}}^{-2} f_{s}$ at $\left(x^{*}, y^{*}\right)=(-0.1,0)$ for all three simulated cases; the vertical dashed lines show the frequency range of wave components in the input packet for each case. Figure 1c shows examples of observed $E^{*}$ for various values of whitecap coverage $W$ and wind speed $U_{10}$ in the vicinity of OWS-P at $50^{\circ} \mathrm{N}, 145^{\circ} \mathrm{W}$ provided by Schwendeman and Thomson (2015) and Thomson et al. (2016). Here we define $H_{\mathrm{eq}}$ as a characteristic breaking wave height given by

$$
H_{\mathrm{eq}}=4 \sqrt{\int_{f_{s}}^{2 f_{s}} E(f) d f} .
$$

The results demonstrate that both the simulated and observed $E^{*}$ have a self-similar shape in the range $f_{s}<$ $f<2 f_{s}$, which is usually called an equilibrium range of a wave spectrum (Phillips 1985). Second, the shape of the simulated wave spectrum for $f>f_{\mathrm{s}}$ is similar to the observations. The $f^{-4}$ dependence in the simulated spectra $E(f)$ is achieved by using the constant steepness spectrum $\left(a_{i} k_{i}=\right.$ const. $)$ in which $E(f) \sim a_{i}^{2} \sim k_{i}^{-2}$ and $k_{i}^{-2} \sim f^{-4}$ from the linear dispersion relation for deep water waves. However, in the field spectra there is much more energy at frequencies $f<f_{\mathrm{s}}$ due to the presence of swell. Many field observations of the speed of visible whitecaps have shown that the dominant speed of breaking waves is about half the phase speed of waves at the peak of the energy spectrum (Melville and Matusov 2002; Gemmrich et al. 2008; Thomson and Jessup 2009; Kleiss and Melville 2010; Schwendeman et al. 2014; Sutherland and Melville 2013). Assuming linear dispersion, this means that the frequency associated with breaking waves $f_{b}$ is noticeably larger than the peak frequency $f_{p}$, and that $f_{b}$ is usually in the equilibrium range. Thus $H_{\mathrm{eq}}$ will be an appropriate choice for a characteristic height of visible breaking waves. Further, since $H_{\text {eq }}$ is not sensitive to the amount of energy of swell waves, which is completely absent in our numerical simulations, it is preferable for the purpose of modelfield observations comparisons of vertical scaling of turbulence dissipation rates. Here, $H_{\text {eq }}$ prior to breaking onset is $0.24,0.20$, and $0.14 \mathrm{~m}$ in the simulated cases 
(a)

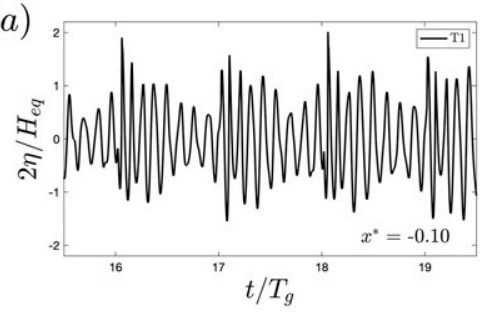

(d)

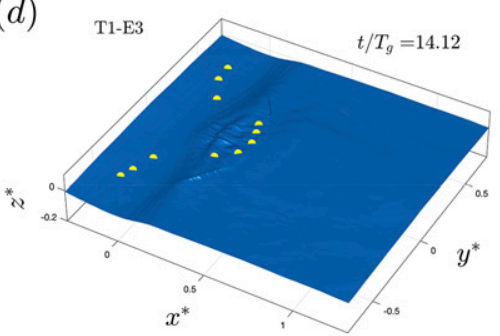

(g)

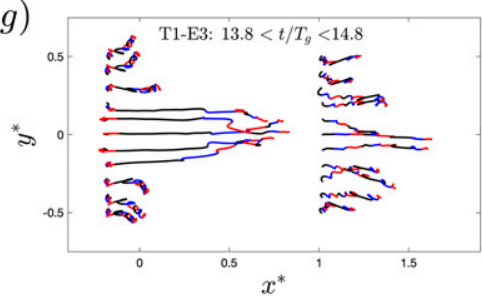

(b)

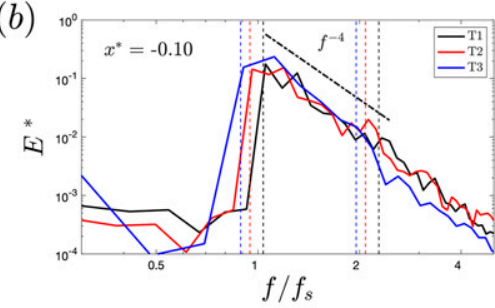

(e)

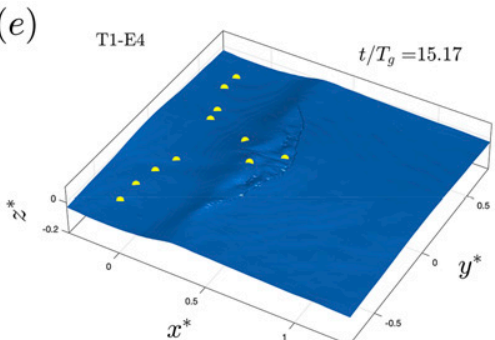

(h)

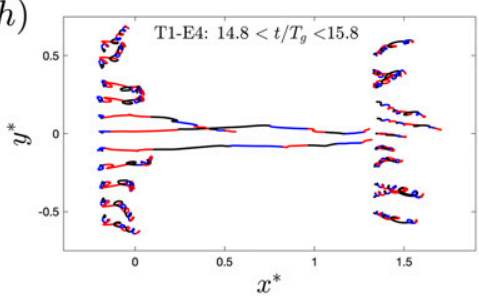

(c)

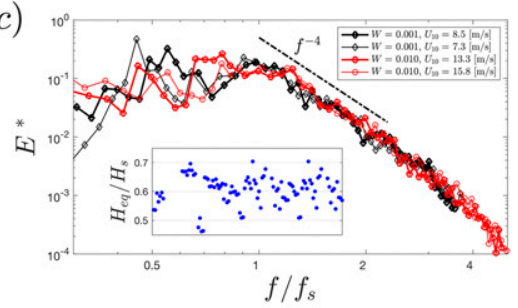

$(f)$

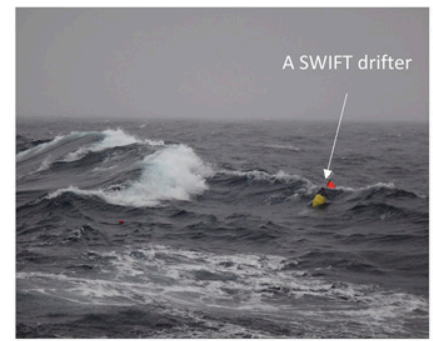

(i)

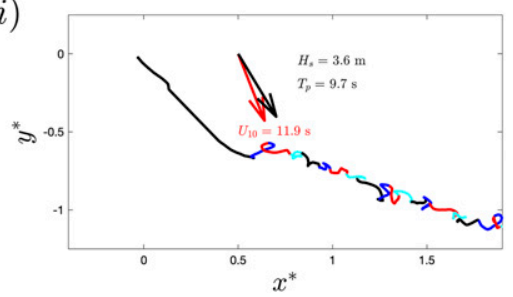

FIG. 1. Comparison between model and field conditions. (a) Temporal variation of the normalized free surface elevations $2 \eta / H_{\mathrm{eq}}$ for the case $\mathrm{T} 1$ and (b) its normalized power spectral density $E^{*}=E H_{\mathrm{eq}}^{-2} f_{s}$ for all three cases at $\left(x^{*}, y^{*}\right)=(-0.1,0)$. (d), (e) Snapshots of the spatial variation of the normalized free surface elevations and of the virtual drifters initially released at $x^{*}=-0.2$ for the breaking events E3 and E4 of the case T1. (g),(h) The normalized horizontal displacements of the virtual drifters shown in (d) and (e). (c),(f),(i) Examples of $E^{*}, H_{\mathrm{eq}} / H_{s}$, a SWIFT drifter, and the horizontal displacement of a SWIFT drifter from the previous field observations in the vicinity of OWS-P at $50^{\circ} \mathrm{N}, 145^{\circ} \mathrm{W}$ (Thomson et al. 2016). Vertical dashed lines in (b) show $f_{1} / f_{s}$ and $f_{N} / f_{s}$, respectively. In (i), the horizontal displacement is measured from the initial location of a drifter and is normalized by the wavelength corresponding to $T_{p} / 2$ ( $\approx 37 \mathrm{~m}$ here), and arrows show the direction of the surface wind and peak wave component. In (g), (h), and (i), each color segment represents the horizontal displacement during $T_{s}$ and $T_{p} / 2$ respectively.

T1, T2, and $\mathrm{T} 3$ respectively, with $H_{\mathrm{eq}} / H_{s} \approx 0.8$ because the main part of the wave energy is distributed in the range $f_{s}<f<2 f_{s}$ (see Fig. 1b).

Figure $1 \mathrm{c}$ shows that $H_{\mathrm{eq}}$ as defined in Eq. (7) varies between 0.45 and 0.7 of the corresponding significant wave height $H_{s}$ for the field conditions with $6<U_{10}<$ $16 \mathrm{~m} \mathrm{~s}^{-1}$. Considering the Pierson-Moskowitz spectrum, we obtain $f_{s}=1.3 f_{p}$ and $H_{\mathrm{eq}} / H_{s}=0.57$, which is consistent with the averaged value of $H_{\mathrm{eq}} / H_{s} \approx 0.6$ obtained from the observations of Schwendeman and Thomson (2015) and Thomson et al. (2016) in the North Pacific.

We define the active breaking index $I(x, y, t)$ as

$$
I(x, y, t)=\mathscr{H}\left[\alpha_{\mathrm{sa}}^{b}(x, y, t)-\alpha_{\mathrm{th}}^{b}\right],
$$

where $\mathscr{H}$ is the Heaviside step function, $\alpha_{\mathrm{sa}}^{b}$ is the vertical average of the bubble void fraction over the surface layer of depth $d_{s}=0.4 H_{\mathrm{eq}}$, and $\alpha_{\mathrm{th}}^{b}$ is a threshold value. Then, we obtain the fraction of the active breaking crests, or active whitecap coverage $W_{A}$, of the breaking events occur between time $t_{1}$ and $t_{2}$ and over the area $\mathscr{b}$ as

$$
W_{A}=\frac{\int_{t_{1}}^{t_{2}} \int_{-y_{1}}^{y_{1}} \int_{x_{1}}^{x_{2}} I d x d y d t}{\not b\left(t_{2}-t_{1}\right)},
$$

where $x_{1}, x_{2}, y_{1}$ indicate the horizontal extent of the averaging area $\mathscr{b}=2 y_{1}\left(x_{2}-x_{1}\right)$ and are set such that it includes the breaking crests. Unless stated otherwise, we set $t_{1}=t_{0}$ and $t_{2}=t_{0}+N_{E} T_{g}$ to do the time averaging over all available breaking events in the virtual tank after $t=t_{0}$. In the field, $W_{A}$ (and $W$ ) is obtained from image processing of visible whitecaps and should be independent of the selected field of view. In Eq. (9), however, $W_{A}$ depends on $\mathscr{A}$. As we will explain later in this paper, choosing $\alpha_{\mathrm{th}}^{b}=0.02$ provides estimates of $W_{A}$ that are consistent with the observed values of whitecap 
coverage in the open ocean (Schwendeman and Thomson 2015). We note that if $d_{s}$ varies between $0.2 H_{\text {eq }}$ and $0.6 H_{\text {eq }}$ the corresponding $W_{A}$ values vary by less than $30 \%$ of the $W_{A}$ value estimated using $d_{s}=0.4 H_{\mathrm{eq}}$ for the simulated cases. Finally, the temporal variation of the instantaneous $W_{A}$ values using Eq. (9) for the various individual simulated breakers (not shown) are consistent with the data reported in Fig. 1 of Callaghan et al. (2016).

\section{d. Conversion of the model results to virtual drifters}

In this paper, our main goal is to examine potential sampling biases and convergence of statistics of the field observations of intermittent wave breaking turbulence collected by surface following platforms (e.g., SWIFT drifters) using our high resolution numerical simulations. To do this, we need to sample our model results, which are available at fixed Eulerian grid points, in a manner which is similar to how a physical drifter (Fig. 1f) obtains samples in the field.

We first introduce a number of virtual drifters that move with the free surface and local liquid velocity in the computational domain. Then, we interpolate the model Eulerian results onto vertical line segments that are attached to the virtual drifters and extend from the instantaneous free surface $z=\eta$ to $z=\eta-l_{\mathrm{vd}}$. In the surface-following reference frame $z_{\mathrm{sf}}=z-\eta$, all the interpolated results will be in the range $-l_{\mathrm{vd}}<z_{\mathrm{sf}}<0$.

For each breaking event $E_{m}\left(m=1,2, \ldots, N_{E}\right)$, a total number of 231 virtual drifters are released at $t=t_{0}+$ $(m-1) T_{g}$, which is well before the onset of the main breaking event $E_{m}$, and remain in the water for a time $T_{g}$. We consider both uniform and random initial spacing of the virtual drifters to make sure that the resultant statistics are independent of the initial deployment of the virtual drifters. Figures $1 \mathrm{~d}$ and $1 \mathrm{e}$ show two snapshots of the instantaneous locations of the virtual drifters (markers) released uniformly at $x^{*}=-0.2,-0.5<y^{*}<0.5$ for the breaking events E3 and E4 of the case T1. Figure $1 \mathrm{f}$ shows a snapshot of a physical drifter in the field in the vicinity of an active breaking crest propagating toward the drifter.

The horizontal location of each virtual drifter is updated using the vertical average of the water horizontal velocity components over the surface layer of depth $0.2 H_{\text {eq }}$. Figures $1 \mathrm{~g}$ and $1 \mathrm{~h}$ show the corresponding horizontal displacements of some of the virtual drifters released in a uniform grid and during the events E3 and E4 of the case T1 respectively. Figure 1i shows an example of the horizontal displacement of a SWIFT drifter in the field. In these frames, each color segment represents the horizontal displacement during a fixed time, equal to $T_{s}$ in the model results and $T_{p} / 2$ in the observations. Both simulated and observed results indicate that a drifter trapped in an active breaking crest may experience horizontal displacements that are significantly greater than when it is riding on a nonbreaking crest. This is consistent with the recent work of Deike et al. (2017b) and Pizzo et al. (2019).

\section{Results}

A glossary of all variables used hereafter is given in Table A1 in the appendix. In our model results, the rate of transfer of energy from the resolved motions to the SGS motions is $\varepsilon_{\mathrm{sgs}}=\nu_{\mathrm{sgs}}|\mathscr{S}|^{2}$ where $\nu_{\mathrm{sgg}}$ is the SGS eddy viscosity, $|\mathscr{S}|^{2}=2 \mathscr{S}_{i j} \mathscr{S}_{i j}$, and $S_{i j}=(1 / 2)\left(\partial \tilde{u}_{i} / \partial x_{j}+\partial \tilde{u}_{j} / \partial x_{i}\right)$ is the resolved rate of strain. The $\varepsilon_{\mathrm{sgs}}$ variable includes both shear- and bubble-induced dissipation (Derakhti and Kirby 2014a). At high Reynolds number, with the filter width much larger than the Kolmogorov length scale, the viscous dissipation rate is typically much smaller than $\varepsilon_{\mathrm{sgs}}$, and thus $\varepsilon_{\mathrm{sgs}}$ approximates the turbulent kinetic energy (TKE) dissipation rate $\varepsilon$, which is commonly considered as a characteristic indicator of intensity of turbulence.

Figure 2 shows two snapshots of 3D variation of $\varepsilon_{\mathrm{sgs}}$ during active breaking period and $\approx 2 T_{s}$ after the breaking-onset for the breaking event E3 of the case T1. Consistent with previous wave breaking simulations (see, e.g., Derakhti and Kirby 2014a, Figs. 11 and 12), our numerical results indicate that wave-breakinginduced $\varepsilon_{\mathrm{sgs}}$ has a strong temporal and spatial variation, with local values of $\varepsilon_{\text {sgs }}$ varying from $O(1) \mathrm{m}^{2} \mathrm{~s}^{-3}$ (or $\mathrm{W} \mathrm{kg}^{-1}$ ) down to the background levels, and with large values of $\varepsilon_{\mathrm{sgs}}$ concentrated near the wave crest and in regions of high void fraction (bubble void fractions are not shown here). The latter is consistent with the recent laboratory measurements of turbulence dissipation rates $\varepsilon$ within wave breaking crests by Deane et al. (2016). They reported large values of $\varepsilon>1 \mathrm{~W} \mathrm{~kg}^{-1}$ during the acoustically active phase of wave breaking in which air is actively entrained and fragmented into bubbles.

As summarized in section 1, in most practical applications the long-time average (e.g., over many wave periods) of TKE dissipation rates over a relatively large surface area, $O\left(100 L_{p} \times 100 L_{p}\right)$, is of interest. In this section, we first examine how the Eulerian averages of $\varepsilon_{\text {sgs }}$ compare with those obtained from surface following virtual drifters. Then we comment on the convergence of statistics obtained from the virtual drifters. Last, we examine the effect of incomplete sampling of $\varepsilon_{\text {sgs }}$ by the virtual drifters due to limited vertical field of view and occlusion due to the entrained bubbles.

\section{a. Lagrangian versus Eulerian averaging of $\varepsilon_{\mathrm{sgs}}$}

Figure 3 a shows the spatiotemporal variation of the horizontal average of $\varepsilon_{\text {sgs }}$ in a surface-following 
(a)

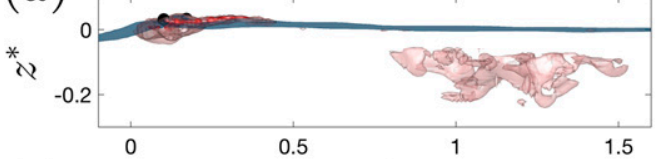

(c)

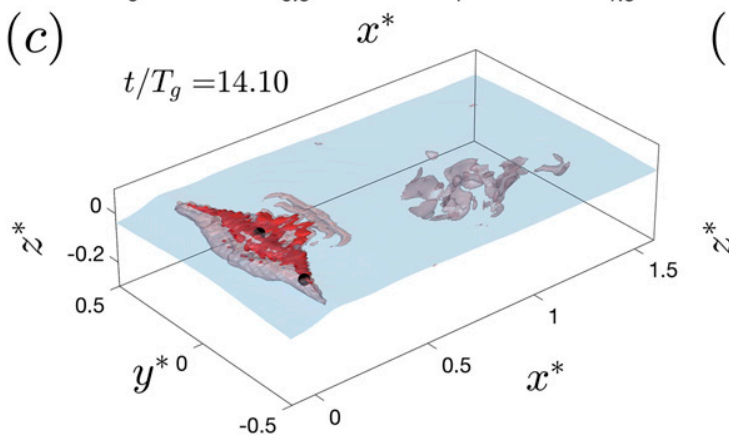

$(b)$

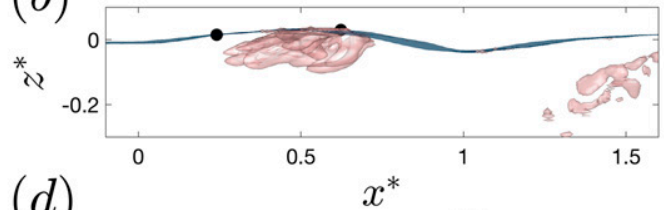

$(d)$

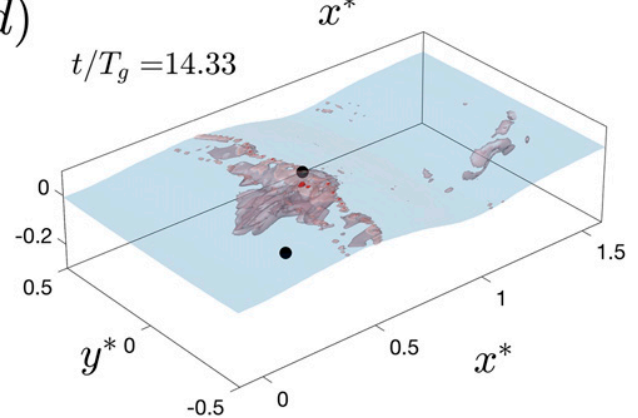

FIG. 2. (a),(b) Side view and (c),(d) 3D view of two snapshots of the spatial variation of the turbulence dissipation rate $\varepsilon_{\mathrm{sgs}}$ for the breaking event E3 of the case T1. Dark and light isosurfaces show $\varepsilon_{\mathrm{sgs}}=0.1$ and $10^{-4} \mathrm{~m}^{2} \mathrm{~s}^{-3}$, respectively. Markers show the location of the two virtual drifters that are initially released at $\left(x^{*}=0, y^{*}=-0.3\right)$ and $\left(x^{*}=0, y^{*}=0\right)$. The waves propagate in the positive $x$ direction. The patches of subsurface $\varepsilon_{\mathrm{sgs}}$ in $x^{*}>0.75$ correspond to the preceding breaking event.

reference frame $\check{\varepsilon}_{\mathrm{sgs}}\left(t, z_{\mathrm{sf}}\right)$ for the breaking event E3 of the case T1. Here, the averaging is performed over a surface that is parallel to $\eta(x, y, t)$ at a distance $\left|z_{\mathrm{sf}}\right|$ below the free surface with the horizontal area of $2 L_{s} \times L_{s}$. The values of $\check{\varepsilon}_{\mathrm{sgs}}$ before the breaking onset $\left(t / T_{s}<133\right)$ and those after $4 T_{s}$ after the breaking onset $\left(t / T_{s}>137\right)$ are approximately comparable, demonstrating that the elevated turbulence dissipation rates due to a wave breaking event return to background levels after a few local wave periods. Figure $3 b$ demonstrates the same trend in the depth integrated values of $\check{\varepsilon}_{\text {sgs }}$. Here the first two peaks are corresponding to the main breaking wave and the successive smaller breaking wave that occur in $133<t / T_{s}<134$ and $134<t / T_{s}<135$, respectively.

Figures $3 \mathrm{c}$ and $3 \mathrm{e}$ show the spatiotemporal variation of $\varepsilon_{\text {sgs }}$ sampled by the two virtual drifters shown in Fig. 2 during E3 of the case T1. Their corresponding timeaveraged profiles, over $T_{g} \approx 9.5 T_{s}$, in the surfacefollowing reference frame are shown by the thick black lines in the Figs. 3d and 3f, where the background thin gray lines represent the results from all available virtual drifters released before the beginning of the breaking event T1-E3. The considerable variation in $\bar{\varepsilon}_{\mathrm{sgs}}$ obtained from virtual drifters indicates that, in the presence of surface wave breaking, the Lagrangian-based values of dissipation rates averaged over approximately 10 wave periods still have a considerable variation depending on the time fraction that a drifter is positioned within the localized turbulence patches generated during the active breaking process. We will examine the convergence of Lagrangian-averaged dissipation rates in the next section.

Figure 4a shows examples of the ensemble-averaged profiles of time-averaged dissipation rate $\overline{\bar{\varepsilon}}^{L}$, which are obtained from the Lagrangian virtual drifters (or timeaveraging over many wave periods at least 1000 wave periods) as

$$
\overline{\bar{\varepsilon}}^{L}=\frac{\sum_{i=1}^{i=231 N_{E}} \bar{\varepsilon}_{\mathrm{sgs}}^{i}}{231 N_{E}} .
$$

Here, $\bar{\varepsilon}_{\mathrm{sgs}}^{i}$ is the time-averaged dissipation rate over time $T_{g}$ sampled by the $i$ th $\left(i=1, \ldots, 231 N_{E}\right)$ virtual drifter (Figs. 3d,f). Figure 4a also shows examples of the ensemble-averaged profiles of the time-horizontalaveraged of the Eulerian model results $\overline{\bar{\varepsilon}}^{E}$ in the surfacefollowing reference frame given by

$$
\overline{\bar{\varepsilon}}^{E}=\frac{\sum_{m=1}^{m=N_{E}} \bar{\varepsilon}_{\mathrm{sgs}}^{m}}{N_{E}},
$$

where $\bar{\varepsilon}_{\mathrm{sgs}}^{m}$ is the time-horizontal-averaged dissipation rate over $T_{g}$ of the breaking event $E_{m}\left(m=1, \ldots, N_{E}\right)$. Further, the corresponding profiles of $\overline{\bar{\varepsilon}}^{E}$ in the fixed reference frame is presented in Fig. $4 \mathrm{~b}$.

The vertical structure of the long-time-averaged turbulence dissipation rate $\overline{\bar{\varepsilon}}$ predicted by the model (Fig. 4) has a number of important features. First our results indicate that $\overline{\bar{\varepsilon}}$ below a surface breaking layer 
$(a)$

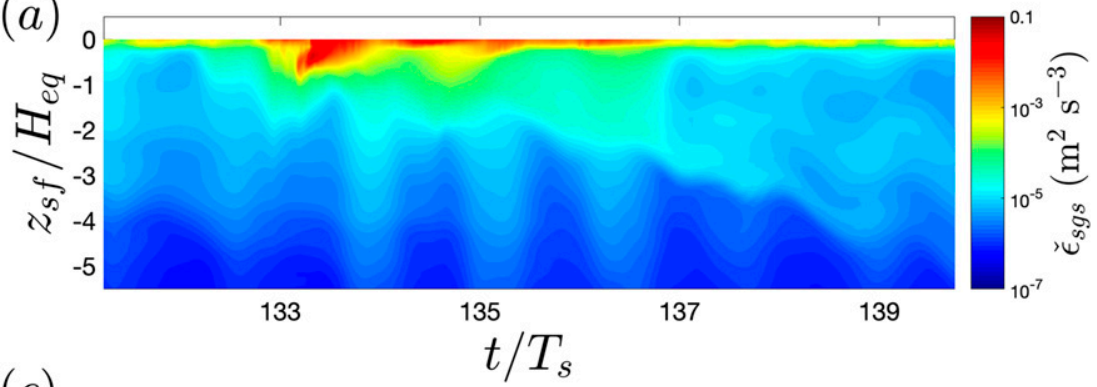

(c)

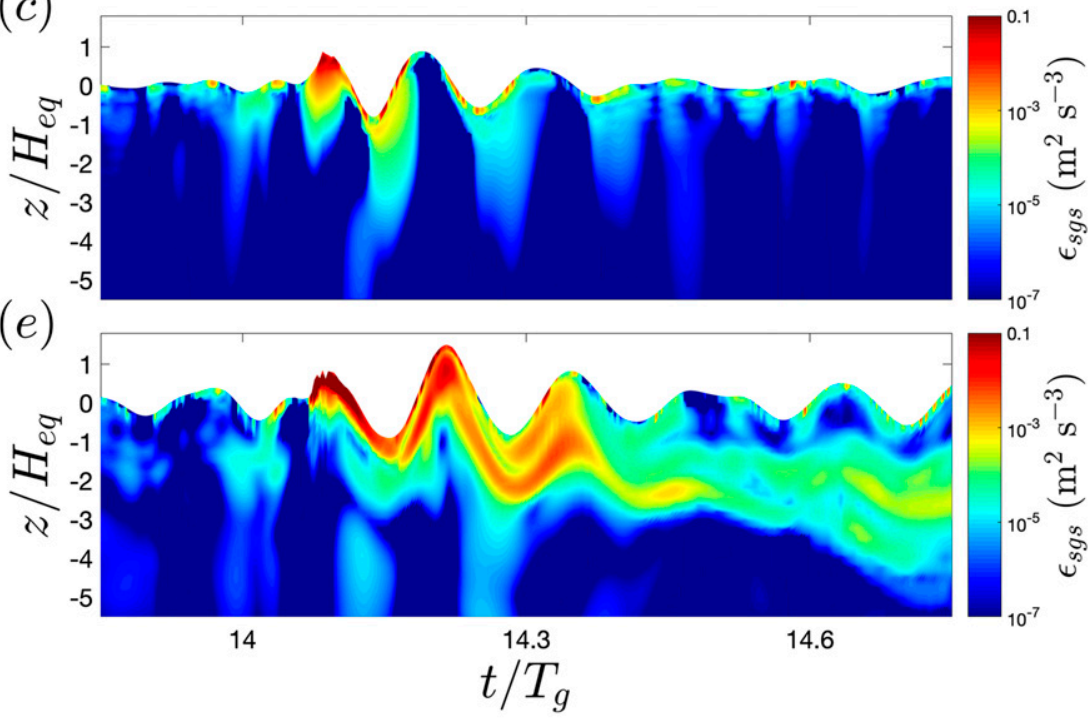

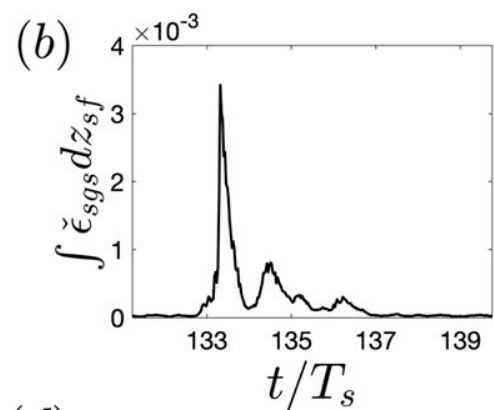

$(d)$

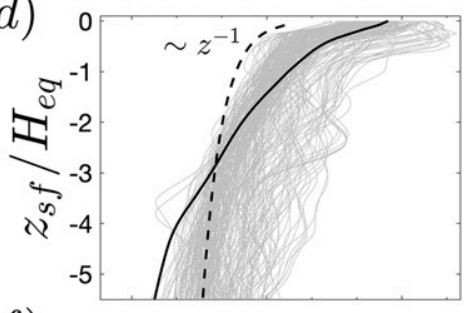

$(f)$

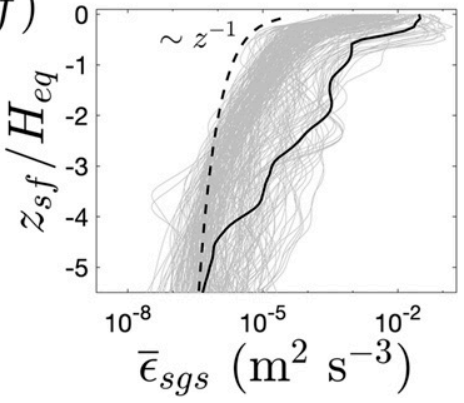

FIG. 3. Various measures of the turbulence dissipation rate $\varepsilon_{\mathrm{sgs}}$ for the breaking event E3 of the case T1. (a) Phase-resolved horizontalaveraged turbulence dissipation rate $\check{\varepsilon}_{\text {sgs }}$ in the surface following reference frame, (b) temporal variation of the vertically integrated $\check{\varepsilon}_{\text {sgs }}$, (c),(e) phase-resolved $\varepsilon_{\text {sgs }}$ sampled by the two virtual drifters shown in Fig. 2, and (d),(f) their corresponding time-averaged vertical profiles $\bar{\varepsilon}_{\mathrm{sgs}}$ (thick black lines) as well as the results for all available virtual drifters released at before the beginning of the breaking event T1-E3 (thin gray lines). The dashed lines in (d) and (f) demonstrate that $\bar{\varepsilon}_{\mathrm{sgs}}$ does not follow the law-of-the-wall vertical scaling $(\sim 1 / z)$.

with depth $H_{\mathrm{eq}}\left(\approx 0.6 H_{s}\right)$ is proportional to $\left(-z_{\mathrm{sf}} / H_{\mathrm{eq}}\right)^{-2}$ in the surface-following reference frame and $\left(-z / H_{\mathrm{eq}}\right)^{-2}$ in the fixed reference frame. This is consistent with the model proposed by Terray et al. (1996) (T96) in the fixed reference frame below the surface breaking layer with depth that T96 took to be $z>0.6 H_{s}$, and with more recent observations in a surface-following reference frame (Gemmrich 2010; Zippel et al. 2018; Sutherland and Melville 2015).

In contrast to T96, but consistent with the same recent field observations (Gemmrich 2010; Sutherland and Melville 2015; Thomson et al. 2016; Zippel et al. 2018), $\overline{\bar{\varepsilon}}$ is not constant in the surface breaking layer $\left(z<0.6 H_{s}\right)$. Our model results indicate that in the upper half of the surface breaking layer in the surface-following reference frame, $\left|z_{s f}\right| / H_{\mathrm{eq}}<1 / 2, \overline{\bar{\varepsilon}}$ is well described with $\overline{\bar{\varepsilon}}\left(z_{\mathrm{sf}}=0\right) \exp \left(\beta z_{\mathrm{sf}} / H_{\mathrm{eq}}\right)$ where $\beta$ is a decreasing function of the active whitecap coverage $W_{A}$. In other words, $\overline{\bar{\varepsilon}}$ will be approximately constant for extreme wave breaking forcing with a relatively large value of $W_{A}$ $(\gg 0.01)$ for $\left|z_{\mathrm{sf}}\right| / H_{\mathrm{eq}}<1 / 2$. Figure $4 \mathrm{a}$ also shows that $\overline{\bar{\varepsilon}}$ still follows the exponential decay in the lower half of the surface breaking layer $1 / 2<\left|z_{\mathrm{sf}}\right| / H_{\mathrm{eq}}<1$ with $\beta$ increasing from approximately 8 for small $W_{A}$ to 9 for large $W_{A}$ values for the simulated cases.

Furthermore, in the fixed reference frame and within the surface breaking layer, $\overline{\bar{\varepsilon}}$ is described with $\overline{\bar{\varepsilon}}(z=0) \exp \left(\beta z / H_{\mathrm{eq}}\right)$ where $\beta$ is again a decreasing function of $W_{A}$ but varies between 3 and 4 for the simulated cases. Our model results demonstrate that the Eulerian maximum of $\overline{\bar{\varepsilon}}$ is above $z=0$, where $\overline{\bar{\varepsilon}}$ has persistently large values in $0<z / H_{\mathrm{eq}}<1$ compared with those in other $z$ elevations. We note that Thomson et al. (2016) have reported a comparable vertical structure for $\overline{\bar{\varepsilon}}$ but with the Eulerian maximum slightly below $z=0$ and the observed values of $\overline{\bar{\varepsilon}}$ in the fixed reference frame immediately started to decrease after reaching their maximum. We suspect that these differences between 

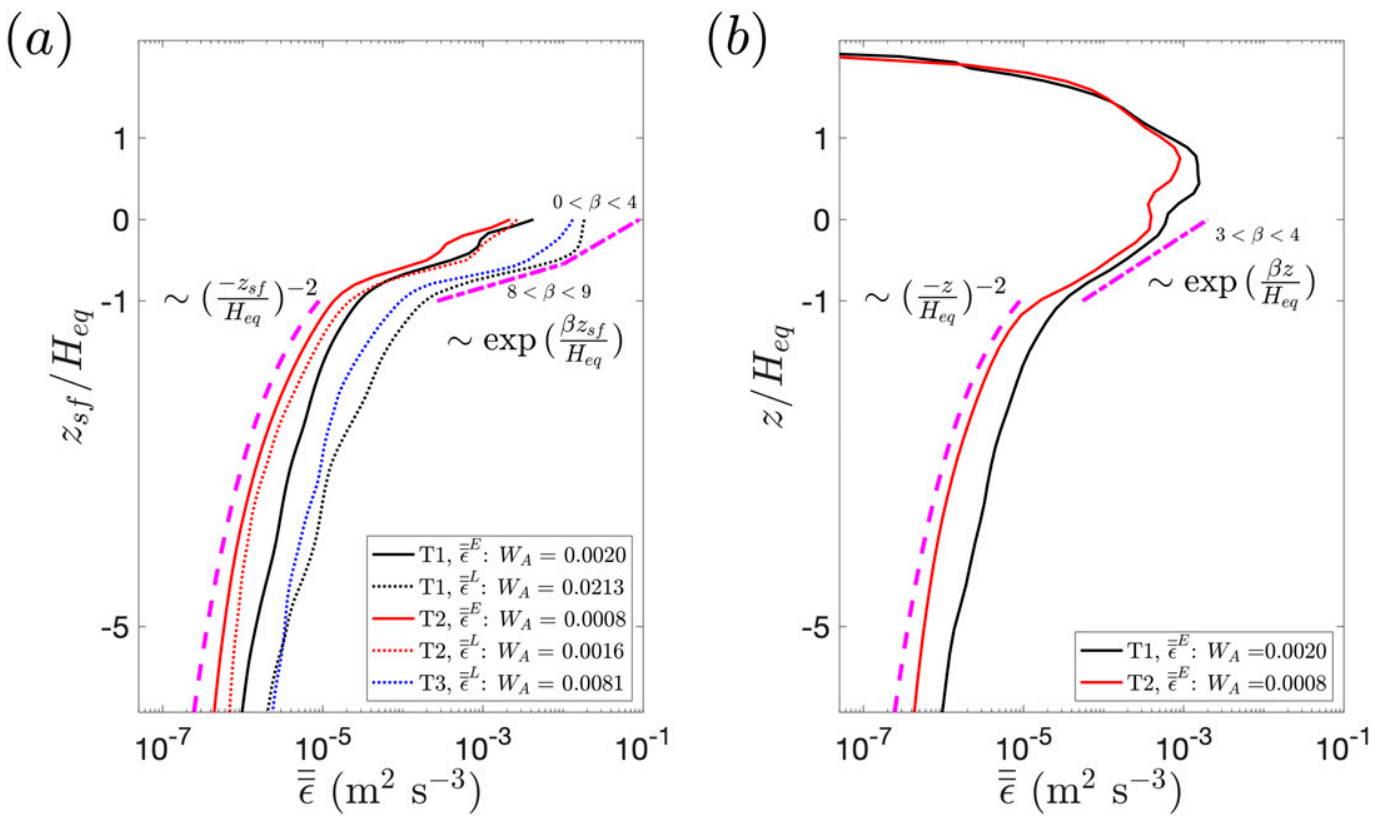

FIG. 4. Model results of the vertical profile of the ensemble-time-averaged turbulence dissipation rates $\overline{\bar{\varepsilon}}$ in (a) the surface-following reference frame and (b) the fixed reference frame. Here $H_{\text {eq }}$ the equilibrium wave height, $W_{A}$ the active whitecap coverage, $\overline{\bar{\varepsilon}}^{L}$, and $\overline{\bar{\varepsilon}}^{E}$ are defined in Eqs. (7), (9), (10), and (11), respectively.

observed and simulated results are mainly due to the limitation of the experimental method in high bubble void fraction regions, and thus, the exclusion of high TKE dissipation rate values in the corresponding data. We will comment on further effects of this issue in section $3 \mathrm{c}$ below.

In addition to the vertical distribution of $\overline{\bar{\varepsilon}}$, the depthintegrated turbulence dissipation rate $\int \overline{\bar{\varepsilon}} d z\left(=\int \overline{\bar{\varepsilon}} d z_{\mathrm{sf}}\right)$ is of significant interest, and as reviewed in section $2 \mathrm{c}$, its relationship with the whitecap coverage $W\left(\right.$ or $W_{A}$ ) remains an active area of research with important impacts for wave prediction modeling, air-sea interaction, and ocean engineering communities. Figure 5a shows the variation of $\int \overline{\bar{\varepsilon}} d z$ with the active whitecap coverage $W_{A}$ [Eq. (9)] for our model results. As reviewed by Brumer et al. (2017), recent observations reveal that $W$ is an increasing function, with the scatter of data, of the wind speed $U_{10}$; varies between $10^{-3}$ and 0.1 for $7<$ $U_{10}<20 \mathrm{~m} \mathrm{~s}^{-1}$. Further, available data of active whitecap coverage $W_{A}=\lambda W(\lambda<1)$ indicates that $\lambda$ is a decreasing function, with the scatter of data, of $U_{10}$; varies between $0.5 \pm 0.25$ and $0.2 \pm 0.1$ for $5<U_{10}<$ $20 \mathrm{~m} \mathrm{~s}^{-1}$ (Scanlon and Ward 2016, Fig. 6b). Thus, the resulting values of $8 \times 10^{-4}<W_{A}<0.021$ shown in Fig. 5a, after dividing by the correction factor $\lambda$, are comparable with the range of observed $W$ values for $7<$ $U_{10}<20 \mathrm{~m} \mathrm{~s}^{-1}$. Thus, we conclude that our definition of $W_{A}$ [Eq. (9)] may be interpreted as an active part of the whitecap coverage values reported in the literature, and that the energetics of wave breaking forcing in the simulated cases may be comparable to most of the available field observations including Schwendeman and Thomson (2015) and Thomson et al. (2016).

Assuming a power-law relationship

$$
\int\left(\overline{\bar{\varepsilon}}-\overline{\bar{\varepsilon}}_{0}\right) d z_{\mathrm{sf}}=a W_{A}^{b}
$$

where $\varepsilon_{0}$ is the background turbulence dissipation rate due to mechanisms other than visible wave breaking, $\varepsilon_{0} \approx 0$ in our simulations. Using least squared curve fitting, we obtain $a=0.077(-0.026,+0.039)$ and $b=0.94( \pm 0.07)$ (with $\left.R^{2}=0.98\right)$, where coefficients in parentheses represent $95 \%$ confidence intervals. This is an interesting and important result, and we will further comment on it in section 4 .

Figure $5 \mathrm{~b}$ shows that a relatively high fraction of total dissipation rate occurs above the mean sea level. This fraction is still noticeably high, $\approx 80 \%$, even for small $W_{A}$ values of about 0.001 . This is consistent with the field observations of Gemmrich (2010) showing that most of the breaking turbulence is concentrated very close to the surface, especially in the wave crest. This is also consistent with the laboratory study of Deane et al. (2016), who found that relatively high dissipation rate values are concentrated in the crest region of the breaking waves. 

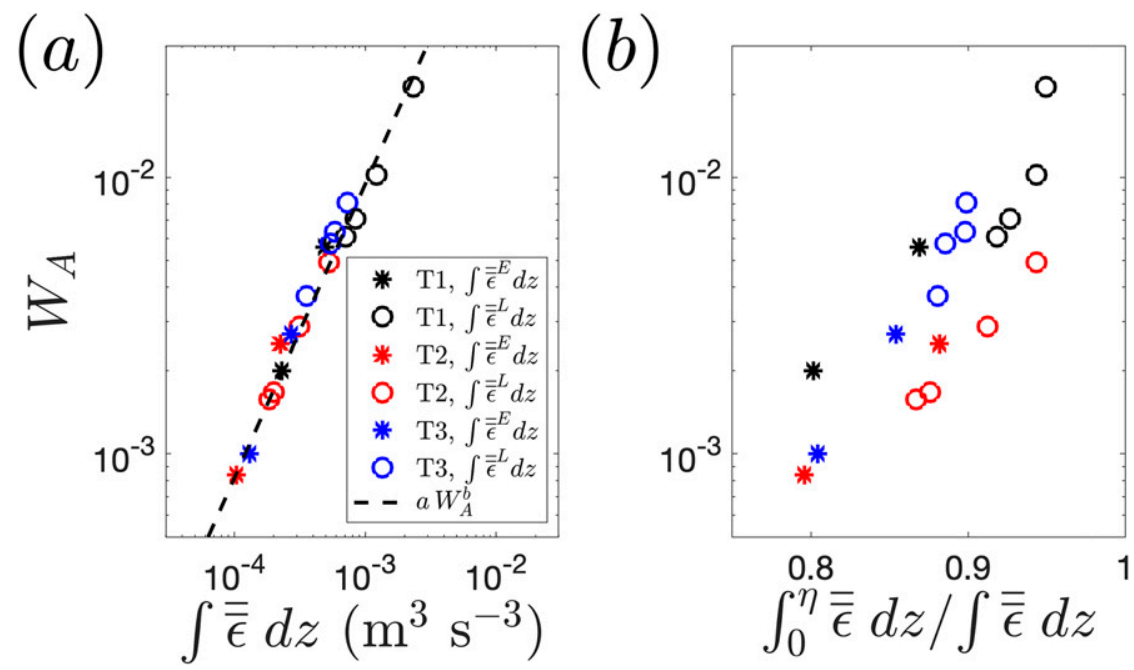

FIG. 5. Model results of the variation of (a) the vertically integrated long-time-averaged dissipation rates, and (b) the fraction of total dissipation above still water level $z=0$ with the active whitecap coverage $W_{A}$ [Eq. (9)].

In particular, Deane et al. (2016) find that the majority of energy dissipation occurs within bubble plumes.

Finally, the results shown in Figs. 4a and 5 demonstrate that the Lagrangian statistics of intermittent wave breaking turbulence, obtained from the sampled data by freely drifting platforms, are representative of the corresponding Eulerian statistics when the length of the Lagrangian data is very large compared with the local wave breaking period. In the next section, we examine how such Lagrangian statistics converge as a function of the length of data.

\section{b. Convergence of statistics}

In this section, we examine how the Lagrangian statistics of dissipation rates obtained from $n$ randomly selected virtual drifters $\overline{\bar{\varepsilon}}_{n}^{L}$ compare to the associated statistics using all available virtual drifters $\overline{\bar{\varepsilon}}^{L}$ [Eq. (10)]. We note that the normalized length of the considered data $\mathscr{L} / T_{s}$ used to obtain $\overline{\bar{\varepsilon}}_{n}^{L}$ and $\overline{\bar{\varepsilon}}^{L}$ are $\approx 9.5 n$ and $\approx 9.5 \times 231 N_{E}$, respectively.

Figure 6 shows $\overline{\bar{\varepsilon}}_{n}^{L}$ (solid lines) for three $n$ values and two different $W_{A}$ as well as the corresponding ensembleaveraged profiles using all the virtual drifters $\overline{\bar{\varepsilon}}_{n}^{L}$ (dashed lines). The random selection of $n$ virtual drifters for each $n$ value was repeated 100 times (gray lines). For better visibility, five examples of these profiles are plotted as individual black curves. As expected for both cases, $\overline{\bar{\varepsilon}}_{n}^{L}$ (solid lines) converges to $\overline{\bar{\varepsilon}}^{L}$ (dashed lines) with increasing $n$ or the length of signal. However, the convergence of statistics with increasing $n$ occurs more rapidly for the case with a larger $W_{A}$ value (or more active breaking) compared with the case with a relatively smaller $W_{A}$ value.
Figure $7 \mathrm{a}$ shows the variation of the normalized RMSE of $\int \overline{\bar{\varepsilon}}_{n}^{L} d z_{\text {sf }}$ (with mean value $\int \overline{\bar{\varepsilon}}^{L} d z_{\text {sf }}$ ) with normalized signal length $\mathscr{L} / T_{s} \approx 9.5 n$ for the two cases shown in Fig. 6. The results show that this error may be fairly reasonably estimated using the Gaussian distribution formulation (dashed lines). Further, Fig. 7b shows the variation of the normalized standard error for four values of $\mathscr{L} / T_{s}=100,500,1000$, and 3000 for all $W_{A}$ values. Our results suggest that the required length of data to obtain stable Lagrangian statistics (with normalized RMSE $<25 \%$ ) of intermittent wavebreaking-induced turbulence using freely drifting platforms should be at least 1000 and 3000 characteristic wave breaking periods for medium to high sea states (e.g., $W_{A}>5 \times 10^{-3}$ ) and low sea states (e.g., $W_{A}<5 \times$ $10^{-3}$ ) respectively. In other words, more Lagrangian sampled data are needed to obtain stable statistics of wave breaking turbulence as the probability of breaking or $W_{A}$ decreases.

Assuming the characteristic wave breaking period of sea waves as $T_{p} / 2$ (see section $2 \mathrm{c}$ ), the minimum required length of data to perform averaging will be $\mathscr{L}_{\min }=500 T_{p}$ and $1500 T_{p}$ for $W_{A}>5 \times 10^{-3}$ and $W_{A}<$ $5 \times 10^{-3}$, respectively. In other words, $\mathscr{L}_{\text {min }}$ varies between 1 and $3 \mathrm{~h}$ depending on a particular sea state. Brumer et al. (2017) and Callaghan and White (2009) found that whitecap data collected over approximately 20-30 $\mathrm{min}$ are needed to reduce uncertainty related to average whitecap coverage values. We note that $\mathscr{L}_{\text {min }}$ may be obtained from one or multiple drifters, with a sampling time of each drifter should be smaller than the time scale during which the wind forcing condition can be assumed as constant. We will show in section 4 that considering 

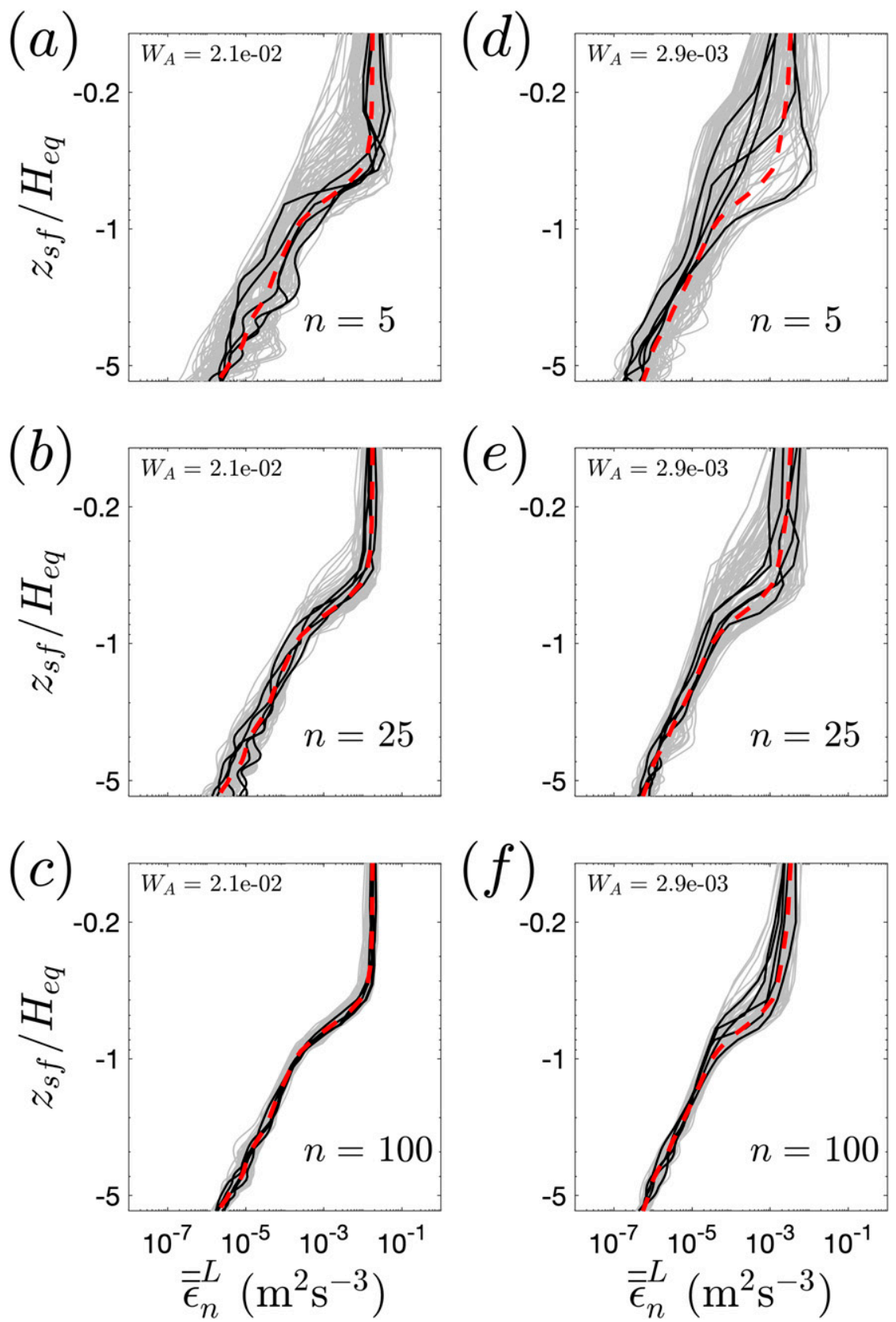

FIG. 6. Variation of the Lagrangian statistics of turbulence dissipation rates obtained from $n$ randomly selected virtual drifters $\overline{\bar{\varepsilon}}_{n}^{L}$ (solid lines) with the signal length $\mathscr{b} \approx 9.5 n T_{s}$ for cases with (a)-(c) $W_{A}=2.1 \times 10^{-2}$, and (d)-(f) $W_{A}=2.9 \times 10^{-3}$. For better visibility, five examples of $\bar{\varepsilon}_{n}^{L}$ profiles are plotted in each frame as individual black curves. The red dashed lines show $\overline{\bar{\varepsilon}}^{L}$, which represents the average of all virtual drifters [Eq. (10)].

$\mathscr{L}_{\text {min }}$ noticeably decreases the amount of scatter in the previous observations of the total TKE dissipation rates.

\section{c. Effects of occlusion due to the entrained bubbles and truncated vertical sampling}

We know from previous numerical (Derakhti and Kirby 2014a) and laboratory (Blenkinsopp and Chaplin
2007) studies that the most active region of turbulence generation and dissipation include relatively large air bubble void fractions. Figure 8a shows the distribution of the number of the simulated data points sampled by the virtual drifters across dissipation rate and bubble void fraction bins, which are uniformly spaced in log scale, for the breaking event E3 of the case T1. The results indicate 

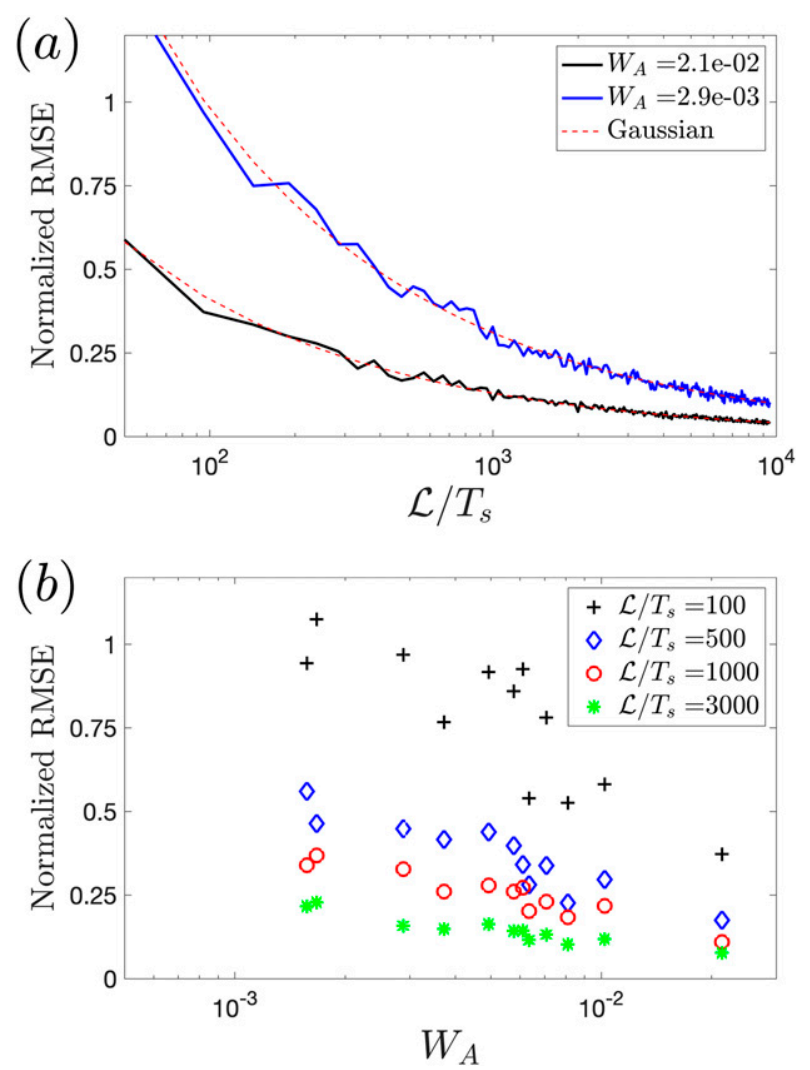

FIG. 7. Variation of the normalized standard error of total turbulence dissipation rate estimates (a) with the signal length $\mathscr{L} / T_{S} \approx 9.5 n$, and (b) with active whitecap coverage $W_{A}$. The dashed lines in (a) show the normalized standard error estimation using the Gaussian distribution.

that $\alpha^{b}>1 \%$ in a noticeable portion of regions with relatively high $\varepsilon_{\text {sgs }}$ values. Figure $8 \mathrm{~b}$ shows examples of the variation of the fraction of the total dissipation within the regions with $\alpha^{b}<\alpha_{0}^{b}$ against $\alpha_{0}^{b}$. In other words, although the high dissipation regions occur in a short portion of time $\left(\sim W_{A}\right)$ but their contribution to the total dissipation is large. The results shown in Fig. 8 reveal that approximately half of the total dissipation occurs in regions with $\alpha^{b}>1 \%$.

Void fractions above $1 \%$ significantly decrease the quality of the data collected by acoustic Doppler methods by decreasing the correlation of coherent pulses (Mori et al. 2007). As a result, a large portion of high dissipation rate values (Fig. 8b) in the observed data are occluded by bubbles. Figure 9a shows the comparison between the vertical profiles of averaged dissipation rates obtained by (solid lines) a regular ensemble-timeaveraging defined in Eq. (10) and (dashed lines) a conditional averaging over data points with $\alpha^{b}<1 \%$ for the two cases with different $W_{A}$ values. Although the effect of the occlusion due to bubbles is limited to

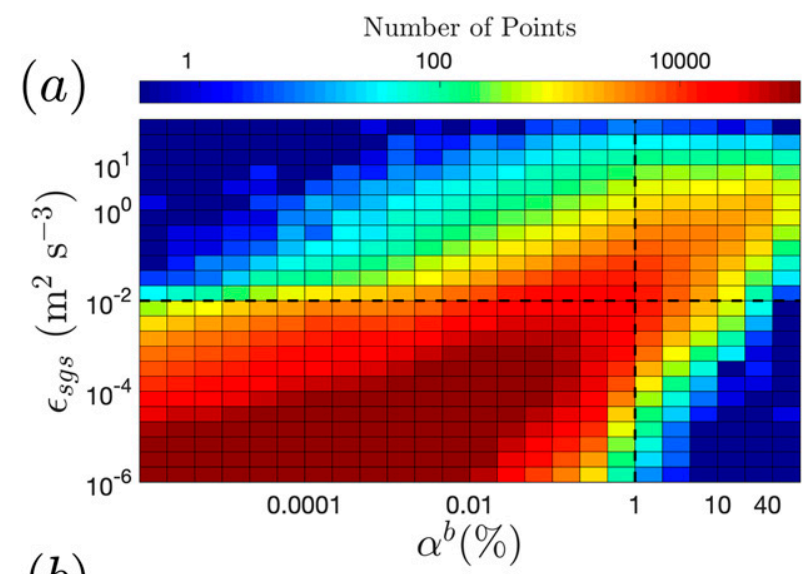

(b)

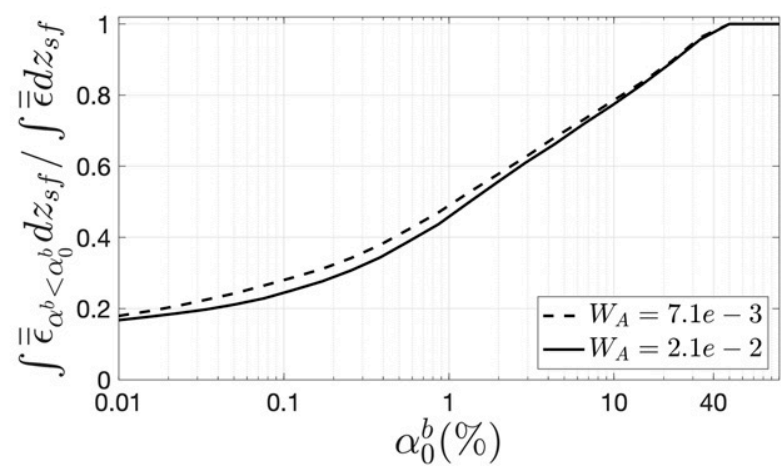

FIG. 8. (a) Example of a 2D histogram of the model results of the local turbulence dissipation rates $\varepsilon_{\text {sgs }}$ and bubble void fraction, and (b) two examples of the variation of the fraction of the total dissipation within the regions with $\alpha^{b}<\alpha_{0}^{b}$ against $\alpha_{0}^{b}$. In (a), the vertical and horizontal dashed lines show $\alpha^{b}=1 \%$ and $\varepsilon_{\text {sgs }}=0.01 \mathrm{~m}^{2} \mathrm{~s}^{-3}$, respectively.

the breaking surface layer $\left|z_{\mathrm{sf}}\right|<0.6 H_{\mathrm{eq}}$, such data occlusion results in a considerable underprediction of the total wave breaking dissipation rates in field observations using acoustic Doppler methods. Further, a limited vertical extend of sampled data by drifters causes the underestimation of the total dissipation rates as well.

If we assume that a drifter can only sample the TKE dissipation rates in regions with $\alpha^{b}<\alpha_{0}^{b}$ and up to a depth $\left|z_{0}\right|$, then the incomplete observed wave-breaking-induced TKE dissipation rates, $\int_{z_{0}}^{0}\left(\overline{\bar{\varepsilon}}-\overline{\bar{\varepsilon}}_{0}\right)_{\alpha^{b}<\alpha_{0}^{b}} d z_{\mathrm{sf}}$, will be always less than their corresponding true values $\int\left(\overline{\bar{\varepsilon}}-\overline{\bar{\varepsilon}}_{0}\right) d z_{\mathrm{sf}}$ [Eq. (12)].

Figure 9b shows examples of the variation of a correction factor $\mathscr{C}>1$ defined as

$$
\mathscr{C}=\frac{\int\left(\overline{\bar{\varepsilon}}-\overline{\bar{\varepsilon}}_{0}\right) d z_{\mathrm{sf}}}{\int_{z_{0}}^{0}\left(\overline{\bar{\varepsilon}}-\overline{\bar{\varepsilon}}_{0}\right)_{\alpha^{b}<\alpha_{0}^{b}} d z_{\mathrm{sf}}},
$$


(a)

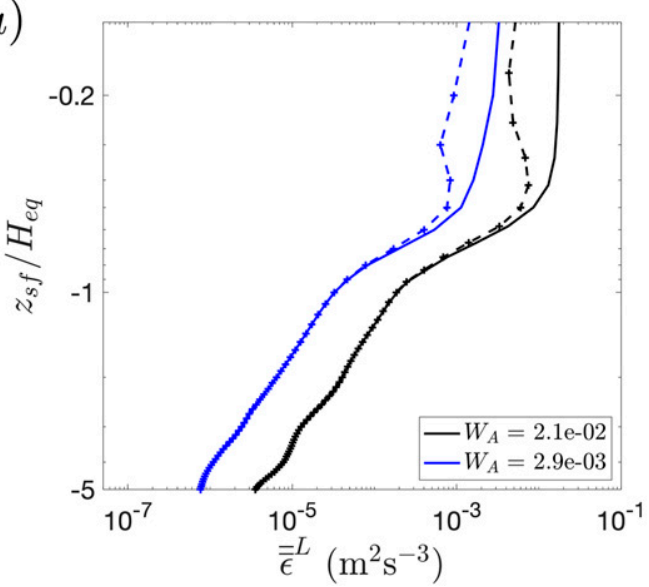

(b)

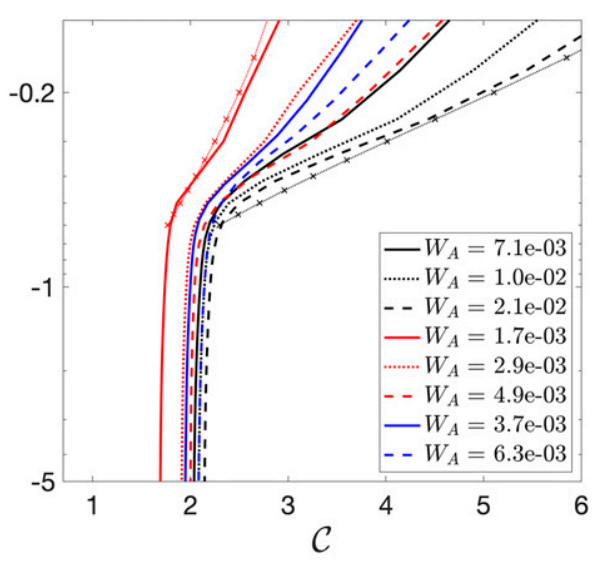

FIG. 9. Incomplete sampling of turbulence dissipation rates. (a) Effect of the bubble occlusion on the Lagrangian averages by considering (solid lines) all model results $\overline{\bar{\varepsilon}}^{L}$ [Eq. (10)], and (dashed lines) only data points in which $\alpha^{b}<1 \%$. (b) Variation of the correction factor $\mathscr{C}$ [Eq. (13)] with depth and the active whitecap coverage $W_{A}$. In (b), the two line segments with markers show the fit defined in Eq. (14) for the smallest and largest $W_{A}$ values of the simulated cases.

with the depth and the active whitecap coverage $W_{A}$ for $\alpha_{0}^{b}=1 \%$. As expected, $\mathscr{C}$ is an increasing function of $W_{A}$ and a decreasing function of $\left|z_{0}\right|$. However, $\mathscr{C}$ has a very weak correlation with $\left|z_{0}\right|$ for $\left|z_{0}\right|>0.6 H_{\text {eq }}$ (or below a breaking surface layer). Based on our numerical results, we obtain a simple relationship to predict $\mathscr{C}$ as a function of $W_{A}$ and $\left|z_{0}\right| / H_{\text {eq }}$ :

$$
\mathscr{C} \approx \mathscr{C}_{b} Z^{\gamma}
$$

where $Z=\operatorname{Min}\left(\left|z_{0}\right| / H_{\mathrm{eq}}, 0.6\right)+0.4$,

$$
\mathscr{C}_{b}=c_{1} \log _{10} W_{A}+c_{2},
$$

and

$$
\gamma=d_{1} \log _{10} W_{A}+d_{2} .
$$

Here the empirical coefficients $c_{1}, c_{2}, d_{1}$, and $d_{2}$ are obtained for a particular choice of $\alpha_{0}^{b}$ and by using the least squared curve fitting. Table 2 documents these parameters for $\alpha_{0}^{b}=(1 / 3) \%, 1 \%$, and $3 \%$.

The two line segments with markers shown in Fig. 9b represent the corresponding fits given in Eqs. (14)-(16) (here $\alpha_{0}^{b}=1 \%$ ) for the smallest and largest $W_{A}$ values of the simulated cases. We conclude that the empirical formulations Eqs. (14)-(16) provide a fairly reasonable approximation for the correction factor $\mathscr{C}$ for the range of $W_{A}$ considered here.

\section{Discussion}

These results (sections $3 \mathrm{~b}$ and $3 \mathrm{c}$ ) improve interpretation of observed long-time-averaged total wave-breaking-induced TKE dissipation rates, $\int\left(\overline{\bar{\varepsilon}}-\overline{\bar{\varepsilon}}_{0}\right) d z_{\mathrm{sf}}$. However, they are purely based on the idealized wave forcing and boundary conditions. Further, there is some uncertainty in generalizing our results to a broader range of field observations, for example, using the correction factor $\mathscr{C}$ [Eqs. (14)-(16)]. Proper application of the model results to field data relies on the connection between the model active whitecap coverage defined in Eq. (9) and field estimates of active whitecap coverage using image processing techniques.

Here, we examine the variation of the corrected total wave-breaking-induced TKE dissipation rates

$$
\int\left(\overline{\bar{\varepsilon}}-\overline{\bar{\varepsilon}}_{0}\right) d z_{\mathrm{sf}} \approx \mathscr{C} \int_{z_{0}}^{0}\left(\overline{\bar{\varepsilon}}-\overline{\bar{\varepsilon}}_{0}\right)_{\mathrm{Obs}} d z_{\mathrm{sf}}
$$

against the corresponding observed whitecap coverage $W$ provided by Schwendeman and Thomson (2015) and the rate of wind energy input examined by Thomson et al. (2016). Schwendeman and Thomson (2015) provided 119 data points including the whitecap coverage $W$ estimated from shipboard video systems, the total observed TKE dissipation rate $\int_{z_{0}}^{0}\left(\overline{\bar{\varepsilon}}-\overline{\bar{\varepsilon}}_{0}\right)_{\mathrm{Obs}} d z_{\mathrm{sf}}$ with $\left|z_{0}\right|=0.42 \mathrm{~m}$, wave spectra parameters, wind speed $U_{10}$,

TABLE 2. The empirical coefficients in Eqs. (15) and (16) for three values of $\alpha_{0}^{b}(\%)$ obtained using the least squared curve fitting. Coefficients in parentheses represent $95 \%$ confidence intervals.

\begin{tabular}{ccccc}
\hline \hline$\alpha_{0}^{b}$ & $c_{1}$ & $c_{2}$ & $d_{1}$ & $d_{2}$ \\
\hline $1 / 3$ & $0.67( \pm 0.21)$ & $4.30( \pm 0.50)$ & $-0.89( \pm 0.15)$ & $-3.00( \pm 0.3)$ \\
1 & $0.48( \pm 0.12)$ & $3.15( \pm 0.26)$ & $-0.79( \pm 0.13)$ & $-2.90( \pm 0.3)$ \\
3 & $0.26( \pm 0.12)$ & $2.20( \pm 0.25)$ & $-0.73( \pm 0.12)$ & $-2.84( \pm 0.3)$ \\
\hline
\end{tabular}


and air-side friction velocity $u_{*}$. Here $\int_{z_{0}}^{0}\left(\overline{\bar{\varepsilon}}-\bar{\varepsilon}_{0}\right)_{\text {Obs }} d z_{\text {sf }}$ is the observed TKE dissipation rate values above a background level and is collected from the freely drifting SWIFT drifters (Thomson 2012). Following the results presented in section $3 \mathrm{~b}$, we also consider clustering of the observed data to obtain averaged values over at least one hour, in addition to applying the correction $\mathscr{C}$ on the observed data.

To estimate $\mathscr{C}$, we need to estimate the active whitecap coverage $W_{A}$ from the observations of $W$ provided by Schwendeman and Thomson (2015), where $W_{A}=\lambda W$ and $\lambda$ ranges from $0.5 \pm 0.25$ to $0.2 \pm 0.1$ in moderate to high sea states (Scanlon and Ward 2016, Fig. 6b). We use a fit, provided by Callaghan (2018), to the bin-averaged $\lambda$ measurements of Scanlon and Ward (2016) given by

$$
\lambda=\frac{1}{1+8.65\left(0.001 U_{10}^{2}+0.02\right)^{0.69}},
$$

in which the estimated $\lambda$ values decreases from $\approx 0.5$ for $U_{10}=5 \mathrm{~m} \mathrm{~s}^{-1}$ to $\approx 0.15$ for $U_{10}=23 \mathrm{~m} \mathrm{~s}^{-1}$ [for further details see Callaghan (2018), Fig. 5a and the related text therein].

\section{a. Observed TKE dissipation rates versus active whitecap coverage}

Figure 10 shows the variation of $\int_{z_{0}}^{0}\left(\overline{\bar{\varepsilon}}-\overline{\bar{\varepsilon}}_{0}\right)_{\mathrm{Obs}} d z_{\mathrm{sf}}$ (denoted by small circles) and $\mathscr{C} \int_{z_{0}}^{0}\left(\overline{\bar{\varepsilon}}-\overline{\bar{\varepsilon}}_{0}\right)_{\mathrm{Obs}} d z_{\mathrm{sf}}$ (denoted by small diamonds) with $W$ (Fig. 10a) and with $W_{A}=\lambda W$ (Fig. 10b) for all available data points, where $\varepsilon_{0}$ represents turbulence dissipation by any mechanisms other than visible whitecaps, for example, microbreakers, Langmuir circulations, internal breaking waves, shear production, and so on. In general, the background turbulence dissipation rate per unit surface area (or $\left.\int\left(\overline{\bar{\varepsilon}}_{0}\right)_{\mathrm{Obs}} d z_{\mathrm{sf}}\right)$ is a sea state dependent quantity, and may vary between $O(0.01)$ (Hwang and Sletten 2008) and $O(0.1) \mathrm{W} \mathrm{m}^{-2}$ (Gemmrich 2010; Sutherland and Melville 2015). Here, we take $\int_{z_{0}}^{0}\left(\overline{\bar{\varepsilon}}_{0}\right)_{\text {Obs }} d z_{\mathrm{sf}}=3.2 \times 10^{-4}\left(\mathrm{~m}^{3} \mathrm{~s}^{-3}\right)$ $\left(\approx 0.3 \mathrm{~W} \mathrm{~m}^{-2}\right)$, which is approximately 0.9 of the minimum observed $\int_{z_{0}}^{0}(\overline{\bar{\varepsilon}})_{\mathrm{Obs}} d z_{\mathrm{sf}}$ value reported by Schwendeman and Thomson (2015). Applying our correction factor $\mathscr{C}$ [Eqs. (14)-(16)] significantly improves the results, such that the variation of the total TKE dissipation rates increases approximately one order of magnitude by increasing $W$ from $\approx 10^{-3}$ to $\approx 3 \times 10^{-2}$. Such strong correlation between $\int\left(\overline{\bar{\varepsilon}}-\overline{\bar{\varepsilon}}_{0}\right) d z_{\text {sf }}$ and $W$, or $W_{A}$, is consistent with our simulation results presented in Fig. 5a and with previous semiempirical and field studies (Hwang and Sletten 2008; Anguelova and Hwang 2016; Callaghan 2018).

Based on the results shown in section $3 \mathrm{~b}$, part of the scatter in the data shown in Fig. 10 (small symbols) may
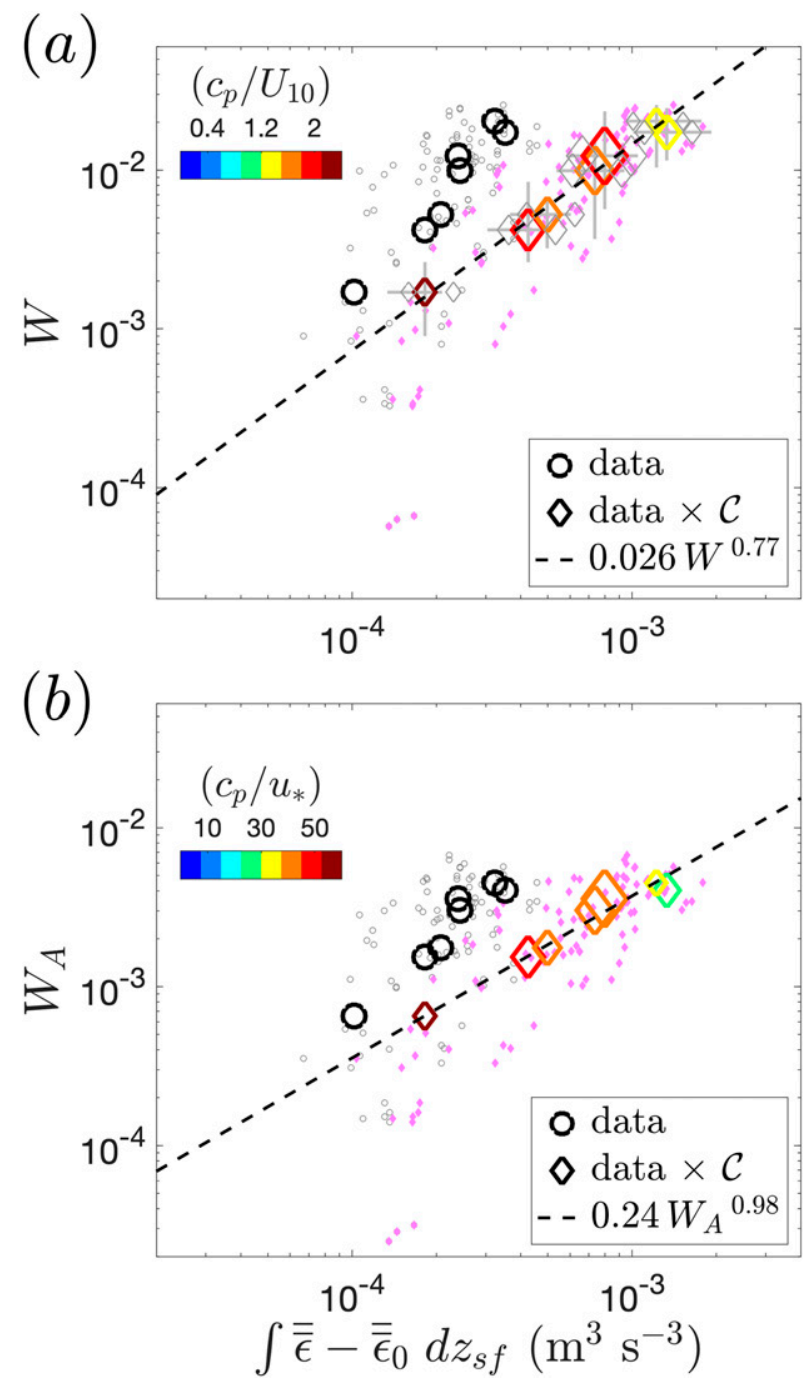

FIG. 10. Variation of the total wave-breaking-induced TKE dissipation rates with (a) total whitecap coverage $W$ and (b) active whitecap coverage $W_{A}=\lambda W$ [Eq. (18)]. Circles show the raw data provided by Schwendeman and Thomson (2015), and diamonds show the corrected results by applying the correction factor $\mathscr{C}$ defined in Eqs. (14)-(16), $\left.\alpha_{0}^{b}=1 \%\right)$. Small and large symbols represent the results considering an averaging time of $\mathscr{C}=512 \mathrm{~s} \approx 40-80 T_{p}$ and those obtained by a conditional clustered-averaging explained in the text with $\mathscr{L}>400 \hat{T}_{p}$. The horizontal line segments represent sensitivity of the results with respect to $0.1<\lambda<0.75$. The small gray diamonds show the sensitivity of the corrected clustered-averaged total dissipation rates with respect to the variation of the resulting correction factor $\mathscr{C}$ for the choice of $\alpha_{0}^{b}=(1 / 3) \%$ and $3 \%$. Vertical line segments represent the range of the observed $W$ values for each bin.

be related to an insufficient record length $\mathscr{L}$ (here $\mathscr{B}=512 \mathrm{~s} \approx 40-80 T_{p}$ ) used to perform the averaging, especially for $W$ values smaller than $5 \times 10^{-3}$. We first sort the data points corresponding to developing $\left(c_{p} \leq U_{10}\right)$ and developed $\left(c_{p}>U_{10}\right)$ sea states into $U_{10}$ bins with a 
spacing of $1 \mathrm{~m} \mathrm{~s}^{-1}$. Then we remove the data points in which the measured $W$ values vary from the threshold power law fit provided by Callaghan et al. (2008) by more than a factor of 3 . Finally, the associated average values of different parameters are calculated using the remaining data points at each bin with enough data points such that $\mathscr{L}>400 \hat{T}_{p}$, hereafter $\widehat{()}$ represents clustered averaging within each $U_{10}$ bin.

Performing the clustered averaging described above on the dataset of Schwendeman and Thomson (2015) results in seven clustered data points with $\hat{U}_{10}=8.2,8.8,10.0,11.2,15.0$, and $15.9 \mathrm{~m} \mathrm{~s}^{-1}$, all characterized as a developed sea state. The corrected and raw clustered averaged values are denoted by large symbols in Fig. 10, where the size and color of the corrected data points represent their relative $\mathscr{L} / \hat{T}_{p}$ and the wave age values respectively. Further, the range of the observed $W$ values for each bin is denoted by the vertical line segment in Fig. 10a. The horizontal line segments represent sensitivity of the results with respect to $0.1<\lambda<0.75$. Finally, the small gray diamonds show the sensitivity of the corrected clusteredaveraged total dissipation rates with respect to the variation of the resulting correction factor $\mathscr{C}$ for the choice of $\alpha_{0}^{b}=(1 / 3) \%$ and $3 \%$.

Using least-squared curve fitting, and assuming a power-law relationship

$$
\int\left(\overline{\bar{\varepsilon}}-\overline{\bar{\varepsilon}}_{0}\right) d z_{\mathrm{sf}}=a_{1}^{\prime} W^{b_{1}^{\prime}}=a_{1} W_{A}^{b_{1}},
$$

we obtain $a_{1}^{\prime}=0.026(-0.012,+0.022)$ and $b_{1}^{\prime}=0.77$ $( \pm 0.12)$ (with $\left.R^{2}=0.98\right)$ and $a_{1}=0.24(-0.17,+0.58)$ and $b_{1}=0.98( \pm 0.2)$ (with $\left.R^{2}=0.97\right)$, where coefficients in parentheses represent $95 \%$ confidence intervals. Both our simulation results (Fig. 5a) and the corrected clustered-averaged observations (Fig. 10b) suggest that the total wave-breaking-induced TKE dissipation rates $\int\left(\overline{\bar{\varepsilon}}-\overline{\bar{\varepsilon}}_{0}\right) d z_{\text {sf }}$ have a power law dependence with $W_{A}$ with the exponent slightly less than 1 . Thus, the empirical relationship between total dissipation and whitecap coverage is approximately linear.

Dynamical explanations for the whitecap coverage dependence are proposed by Callaghan (2018), who scales dissipation rates with the volume of bubble plumes caused by breaking waves (and thereby the active whitecap coverage and bubble plume penetration depth). In particular, results with a fixed averaged bubble penetration depth in Fig. 8 of Callaghan (2018) show a similar dependence in comparison to Figs. 5a and 10b in the present work. Anguelova and Hwang (2016) also demonstrate a relation between active whitecap coverage and total wave breaking dissipation rates. We further comment on this in the next section. Quantification of averaged penetration depth of bubble plumes relative to active whitecap areas is left for future study.

\section{b. Dissipation scaling and the distribution of breaking crests}

Many previous studies (e.g., Melville and Matusov 2002; Thomson and Jessup 2009; Romero et al. 2012; Sutherland and Melville 2013) have applied the Phillips (1985) framework to estimate an energy dissipation rate using the fifth moment of the breaking crest-length distribution $\Lambda(c)$, where $c$ is the crest speed, as

$$
\int\left(\overline{\bar{\varepsilon}}-\overline{\bar{\varepsilon}}_{0}\right) d z_{\mathrm{sf}}=\frac{\rho b}{g} \int c^{5} \Lambda(c) d c .
$$

Although the present study lacks measurements of $\Lambda(c)$, the results herein are still relevant to the scaling of the breaking dissipation rate and the breaking strength parameter $b$. Beginning with the laboratory work of Drazen et al. (2008), the emerging literature suggests a dependence $b \sim(A k)^{5 / 2}$, where $A k$ is the wave steepness given by amplitude $A$ and wavenumber $k$. Romero et al. (2012) extended this from the steepness of wave packets in the laboratory to the spectral steepness, such that dissipation could be prescribed in a spectral wave model. Zappa et al. (2016) recently reviewed the published results on the breaking strength parameter $b$.

Although we do not evaluate a spectral dissipation rate or the breaking strength with the present analysis, we can attempt to reconcile the $\Lambda(c)$ framework with the relationship between the total dissipation rate and active whitecap coverage. Kleiss and Melville (2010) relate active whitecap coverage $W_{A}$ to the first moment of $\Lambda(c)$ and a time scale for the persistence of the breaking crest $\tau$,

$$
W_{A}=\int c \tau \Lambda(c) d c .
$$

If the time scale $\tau$ is proportional to breaking wave period $T$, then dispersion implies it is proportional to phase speed and the effective relation is active whitecap coverage and the second moment, $W_{A}=\int c^{2} \Lambda(c) d c$. Kleiss and Melville (2010) evaluated these formulations, along with the zeroth moment of $\Lambda(c)$, and find that all have strong linear relations $\left(R^{2}>0.96\right)$ to the observed active whitecap coverage (their Fig. 7). If these lower moments of $\Lambda(c)$ are all similarly related to $W_{A}$, we can expect higher moments to be related as well. Generally, for a narrow distribution, higher moments have a quasilinear relationship to lower moments, because the tail of the distribution is sufficiently small as to have minimal effect. Given the canonical tail of $\Lambda(c) \sim c^{-6}$ 
(Melville and Matusov 2002), the distribution is indeed narrow and the net dependence of total dissipation is closer to $c^{-1}$.

The implications for spectral dissipation remain to be determined, but it is thus at least empirically consistent for both active whitecap coverage $W_{A}$ (Figs. 5a and 10b) and $\int c^{5} \Lambda(c) d c$ to be related to the total wave breaking dissipation rate.

\section{c. Observed TKE dissipation versus wind energy input rates}

In an equilibrium sea state, the rate of wind energy input per unit area to the upper ocean $F\left(\mathrm{~m}^{3} \mathrm{~s}^{-3}\right.$ or $\mathrm{W} \mathrm{kg}^{-1}$ ) is balanced mainly by the wave breaking energy dissipation. Figure 11 demonstrates the significance of applying our correction factor $\mathscr{C}$ to the data from Thomson et al. (2016) in observing this expected equilibrium balance. In addition, the results show that clustering of the individual data points (to achieve long enough record lengths $\mathscr{L}$ ) noticeably reduces the scatter. Here, we use the formulation $F=c_{e} u_{*}^{2} \rho^{a} / \rho$, where $\rho^{a}$ is a constant air density. Other formulations for the wind input rate give similar results (see Thomson et al. 2016). The vertical and horizontal line segments represent sensitivity of the results with respect to $2<c_{e}<3$ and $0.1<\lambda<0.75$, respectively. As in Fig. 10, the small gray diamonds show the sensitivity of the corrected clustered-averaged total dissipation rates with respect to the variation of the resulting correction factor $\mathscr{C}$ for the choice of $\alpha_{0}^{b}=(1 / 3) \%$ and $3 \%$.

Given equilibrium conditions in which wind input and breaking dissipation rates balance, it is not surprising that whitecap coverage has a nearly linear relationship to dissipation rate. Both whitecap coverage and wind input have been regularly related to the cube of the wind speed (e.g., Brumer et al. 2017) or the cube of wind friction velocity (e.g., Craig and Banner 1994). The implied empirical dependence between these parameters is thus linear, with dynamic interpretation still an open question.

\section{Summary}

A high-resolution two-fluid LES/VOF numerical model (Derakhti and Kirby 2014a) representing breaking waves and turbulence is used to show that robust estimates of average turbulence dissipation rates are possible from sparse Lagrangian sampling in a surfacefollowing reference frame (as done with field observations). Bubbles are treated as a multicomponent continuum, with different components representing different bubble diameters. Turbulence is modeled using LES with a dynamic Smagorinsky closure. Bubble contributions

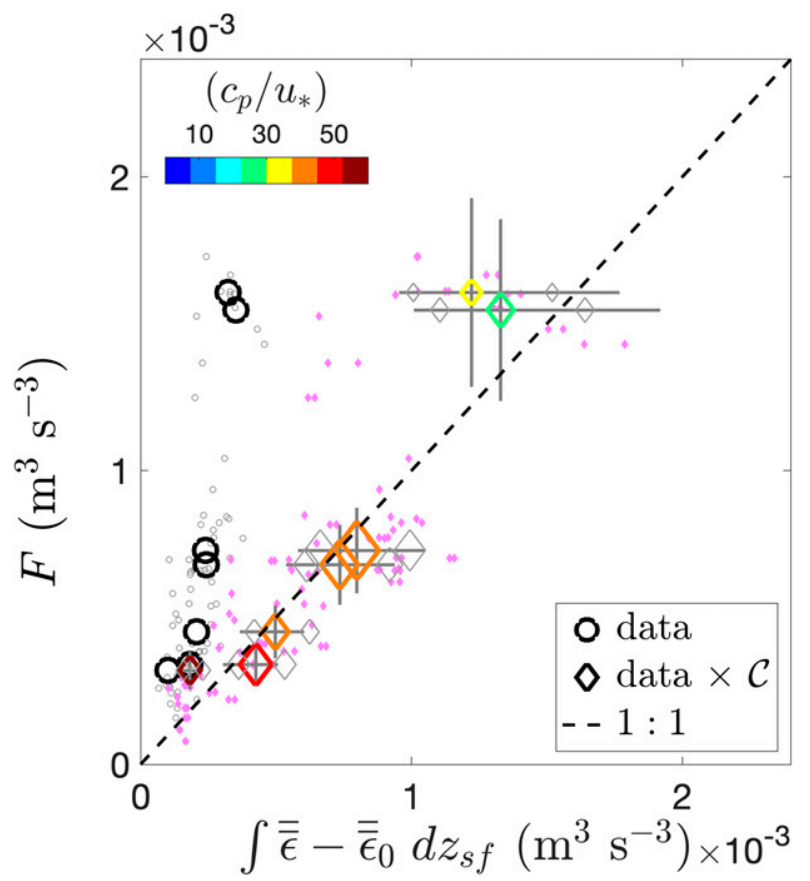

FIG. 11. Variation of the total wave-breaking-induced TKE dissipation rates with the rate of wind energy input $F$. Vertical line segments represent the sensitivity of $F$ values with respect to $2<$ $c_{e}<3$. Definitions of the rest of symbols and lines are the same as in Fig. 10.

to dissipation and momentum transfer between the water and air phases are considered. Numerical simulations are run for many wave periods to build up quasisteady background turbulence levels, with breaking events occurring approximately every $10 T$, where $T$ is the wave period. We sample the LES/VOF model results with a large number of virtual surface-following drifters that are initially distributed in the numerical domain, regularly or irregularly, before each breaking event. Time-averaged Lagrangian statistics are obtained using the time series sampled by the virtual drifters.

Convergence of statistics occurs for signals that have a minimum length of approximately $1000 T$ with randomly spaced observations in time and space relative to $3 \mathrm{D}$ breaking events. This result holds over a wide range of relative breaking activity, which is scaled in the model domain to match field observations of whitecap coverage. The model results also indicated that the high turbulence dissipation rates are correlated with bubble plumes (and thus high void fractions). Using a canonical cutoff of 0.01 void fraction $\left(\alpha^{b}=1 \%\right)$ for field observations of turbulence, an empirical correction factor $\mathscr{C}=\mathscr{C}\left(W_{A},\left|z_{0}\right| / H_{\mathrm{eq}}\right)$ is developed and applied to the previous observations of Thomson et al. (2016), where $W_{A}$ is the active whitecap coverage, $\left|z_{0}\right|$ is the extent of 
TABLE A1. Summary of mathematical variables and symbols used in sections 3-5. Here the dash (-) indicates that the corresponding variable is dimensionless. The order of the symbols is consistent with the order of their first appearance in sections 3-5.

\begin{tabular}{|c|c|c|}
\hline Symbol & Definition & Units \\
\hline$\varepsilon$ & TKE dissipation rate & $\mathrm{m}^{2} \mathrm{~s}^{-3}$ \\
\hline$\nu_{\mathrm{sgs}}$ & SGS eddy viscosity & $\mathrm{m}^{2} \mathrm{~s}^{-1}$ \\
\hline$\varepsilon_{\mathrm{sgs}}$ & Energy transfer rate from the resolved to SGS motions & $\mathrm{m}^{2} \mathrm{~s}^{-3}$ \\
\hline$\check{\varepsilon}_{\text {sgs }}$ & Horizontal average of $\varepsilon_{\mathrm{sgs}}$ in a surface-following reference frame & $\mathrm{m}^{2} \mathrm{~s}^{-3}$ \\
\hline $\bar{\varepsilon}_{\mathrm{sgs}}$ & Time-averaged $\varepsilon_{\mathrm{sgs}}$ over a few wave periods obtained from one Lagrangian virtual drifter & $\mathrm{m}^{2} \mathrm{~s}^{-3}$ \\
\hline $\bar{\varepsilon}^{L}$ & Time-averaged $\varepsilon_{\text {sgs }}$ over many wave periods obtained from all the Lagrangian virtual drifters & $\mathrm{m}^{2} \mathrm{~s}^{-3}$ \\
\hline$\overline{\bar{\varepsilon}}_{n}^{L}$ & Time-averaged $\varepsilon_{\mathrm{sgs}}$ obtained from $n$ randomly selected Lagrangian virtual drifters & $\mathrm{m}^{2} \mathrm{~s}^{-3}$ \\
\hline$\overline{\bar{\varepsilon}}^{E}$ & Time-averaged $\check{\varepsilon}_{\text {sgs }}$ over many wave periods & $\mathrm{m}^{2} \mathrm{~s}^{-3}$ \\
\hline$f_{s}$ & Spectrally weighted frequency of a wave field [Eq. (6)] & $\mathrm{s}^{-1}$ \\
\hline$f_{p}$ & Peak frequency of a wave field & $\mathrm{s}^{-1}$ \\
\hline$T_{s}$ & $=1 / f_{s}$ & $\mathrm{~s}$ \\
\hline$T_{p}$ & $=1 / f_{p}$ & s \\
\hline$T_{g}$ & Wave packet period & s \\
\hline$L_{s}$ & Characteristic wavelength calculated based on $f_{s}$ & $\mathrm{~m}$ \\
\hline$L_{p}$ & Characteristic wavelength calculated based on $f_{p}$ & $\mathrm{~s}$ \\
\hline$\eta$ & Free surface elevation & $\mathrm{m}$ \\
\hline$z_{\mathrm{sf}}$ & Vertical elevation in a surface-following reference frame & $\mathrm{m}$ \\
\hline$H_{s}$ & Significant wave height & $\mathrm{m}$ \\
\hline$H_{\mathrm{eq}}$ & Characteristic wave breaking height [Eq. (7)] & $\mathrm{m}$ \\
\hline$W$ & Whitecap coverage & - \\
\hline$W_{A}$ & Active whitecap coverage & - \\
\hline$\lambda$ & $=W_{A} / W$ & - \\
\hline$U_{10}$ & 10-m wind speed & $\mathrm{m} \mathrm{s}^{-1}$ \\
\hline $\int \overline{\bar{\varepsilon}} d z_{\mathrm{sf}}$ & $=\int \overline{\bar{\varepsilon}} d z$, total energy dissipation rate & $\mathrm{m}^{3} \mathrm{~s}^{-3}$ \\
\hline$\overline{\bar{\varepsilon}}_{0} d z_{\mathrm{sf}}$ & Total energy dissipation rate due to background processes & $\mathrm{m}^{3} \mathrm{~s}^{-3}$ \\
\hline $\mathscr{L}$ & Record length & $\mathrm{s}$ \\
\hline$\alpha^{b}$ & Bubble void fraction & - \\
\hline$\alpha_{0}^{b}$ & Bubble void fraction threshold above which the data is assumed to be occluded by bubbles & - \\
\hline $\mathscr{C}$ & The correction factor defined in Eq. (13) & - \\
\hline$\hat{T}_{p}$ & Clustered averaged $T_{p}$ within $U_{10}$ bins & $\mathrm{s}$ \\
\hline$\hat{U}_{10}$ & Clustered averaged $U_{10}$ & $\mathrm{~m} \mathrm{~s}^{-1}$ \\
\hline$\hat{u}_{*}$ & Air side friction velocity & $\mathrm{m} \mathrm{s}^{-1}$ \\
\hline$c_{p}$ & $=L_{p} / T_{p}$ & $\mathrm{~m} \mathrm{~s}^{-1}$ \\
\hline$c$ & Wave phase speed & $\mathrm{m} \mathrm{s}^{-1}$ \\
\hline$b$ & Breaking strength parameter & - \\
\hline$A k$ & Wave steepness & - \\
\hline$\Lambda(c)$ & Distribution of the breaking wave crest lengths per unit sea surface area per unit increment in velocity $c$ & $\mathrm{~m}^{-2} \mathrm{~s}$ \\
\hline$F$ & Wind energy input rate & $\mathrm{m}^{3} \mathrm{~s}^{-3}$ \\
\hline
\end{tabular}

measurements over depth, and $H_{\mathrm{eq}}$ is a characteristic breaking wave height.

Applying the correction factor to observations significantly alters the estimations of average turbulence dissipation rates sampled by surface following drifters, especially in high sea states, and thus, improves the inferred energy balance of wind input rates and turbulence dissipation rates. Finally, both our simulation results and the corrected observations suggested that the total wave breaking dissipation rates have a nearly linear relation with active whitecap coverage.

We emphasize that the proposed correction factor is based purely on numerical simulations of a limited number of idealized wave breaking events, in which a number of relevant processes such as direct wind forcing have been ignored. In the absence of new field methods for direct observation of turbulence inside bubble plumes, applying the proposed correction factor to the open ocean conditions must be made cautiously. More field observations of near-surface turbulence and bubble plumes are needed, especially in high sea states.

Acknowledgments. This work was supported by Grants OCE-1756040 and OCE-1756355 from the U.S. National Science Foundation. SWIFT data are available from www.apl.washington.edu/swift and whitecap coverage data are available from http://hdl.handle.net/1773/42596. 


\section{APPENDIX}

\section{A Summary of All Mathematical Variables and Symbols Used in Sections 3-5}

Table A1 summarizes the symbols, definitions, and units for the variables used in the results, discussion, and summary sections.

\section{REFERENCES}

Agrawal, Y. C., E. A. Terray, M. A. Donelan, P. A. Hwang, A. J. Williams III, W. M. Drennan, K. K. Kahma, and S. A. Krtaigorodskii, 1992: Enhanced dissipation of kinetic energy beneath surface waves. Nature, 359, 219-220, https://doi.org/ 10.1038/359219a0.

Anguelova, M. D., and P. A. Hwang, 2016: Using energy dissipation rate to obtain active whitecap fraction. J. Phys. Oceanogr., 46, 461-481, https://doi.org/10.1175/JPO-D-15-0069.1.

Babanin, A. V., 2011: Breaking and Dissipation of Ocean Surface Waves. Cambridge University Press, $480 \mathrm{pp}$.

Blenkinsopp, C. E., and J. R. Chaplin, 2007: Void fraction measurements in breaking waves. Proc. Roy. Soc. London, 463A, 3151-3170, https://doi.org/10.1098/rspa.2007.1901.

Brumer, S. E., C. J. Zappa, I. M. Brooks, H. Tamura, S. M. Brown, B. W. Blomquist, C. W. Fairall, and A. Cifuentes-Lorenzen, 2017: Whitecap coverage dependence on wind and wave statistics as observed during SO GasEx and HiWinGS. J. Phys. Oceanogr., 47, 2211-2235, https://doi.org/10.1175/JPO-D17-0005.1.

Callaghan, A. H., 2018: On the relationship between the energy dissipation rate of surface-breaking waves and oceanic whitecap coverage. J. Phys. Oceanogr., 48, 2609-2626, https:// doi.org/10.1175/JPO-D-17-0124.1.

, and M. White, 2009: Automated processing of sea surface images for the determination of whitecap coverage. J. Atmos. Oceanic Technol., 26, 383-394, https://doi.org/ 10.1175/2008JTECHO634.1.

—_, G. B. Deane, and M. D. Stokes, 2016: Laboratory airentraining breaking waves: Imaging visible foam signatures to estimate energy dissipation. Geophys. Res. Lett., 43, 11320-11 328, https://doi.org/10.1002/2016GL071226.

$\ldots, \ldots$, and -2017 : On the imprint of surfactant-driven stabilization of laboratory breaking wave foam with comparison to oceanic whitecaps. J. Geophys. Res. Oceans, 122, 6110-6128, https://doi.org/10.1002/2017JC012809.

—_, G. de Leeuw, L. Cohen, and C. D. O'Dowd, 2008: Relationship of oceanic whitecap coverage to wind speed and wind history. Geophys. Res. Lett., 35, L23609, https:// doi.org/10.1029/2008GL036165.

Carrica, P. M., D. Drew, F. Bonetto, and R. T. Lahey, 1999: A polydisperse model for bubbly two-phase flow around a surface ship. Int. J. Multiphase Flow, 25, 257-305, https://doi.org/ 10.1016/S0301-9322(98)00047-0.

Craig, P. D., and M. L. Banner, 1994: Modeling wave-enhanced turbulence in the ocean surface layer. J. Phys. Oceanogr., 24, 2546-2559, https://doi.org/10.1175/1520-0485(1994)024<2546: MWETIT $>2.0 . C O ; 2$.

Deane, G. B., M. D. Stokes, and A. H. Callaghan, 2016: The saturation of fluid turbulence in breaking laboratory waves and implications for whitecaps. J. Phys. Oceanogr., 46, 975-992, https://doi.org/10.1175/JPO-D-14-0187.1.
Deike, L., W. K. Melville, and S. Popinet, 2016: Air entrainment and bubble statistics in breaking waves. J. Fluid Mech., 801, 91-129, https://doi.org/10.1017/jfm.2016.372.

_ , L. Lenain, and W. K. Melville, 2017a: Air entrainment by breaking waves. Geophys. Res. Lett., 44, 3779-3787, https:// doi.org/10.1002/2017GL072883.

— N. Pizzo, and W. K. Melville, 2017b: Lagrangian transport by breaking surface waves. J. Fluid Mech., 829, 364-391, https:// doi.org/10.1017/jfm.2017.548.

Derakhti, M., and J. T. Kirby, 2014a: Bubble entrainment and liquid bubble interaction under unsteady breaking waves. J. Fluid Mech., 761, 464-506, https://doi.org/10.1017/jfm.2014.637.

— , and — 2014b: Bubble entrainment and liquid bubble interaction under unsteady breaking waves. Research Rep. CACR-14-06, Center for Applied Coastal Research, University of Delaware, Newark, DE, 106 pp., http://www.udel.edu/kirby/ papers/derakhti-kirby-cacr-14-06.pdf.

$\longrightarrow$, and —, 2016: Breaking-onset, energy and momentum flux in unsteady focused wave packets. J. Fluid Mech., 790, 553-581, https://doi.org/10.1017/jfm.2016.17.

— M. L. Banner, and J. T. Kirby, 2018: Predicting the breaking strength of gravity water waves in deep and intermediate depth. J. Fluid Mech., 848, R2, https://doi.org/10.1017/jfm.2018.352.

Drazen, D., W. K. Melville, and L. Lenain, 2008: Inertial scaling of dissipation in unsteady breaking waves. J. Fluid Mech., 611, 307-332, https://doi.org/10.1017/S0022112008002826.

Esters, L., Ø. Breivik, S. Landwehr, A. ten Doeschate, G. Sutherland, K. H. Christensen, J.-R. Bidlot, and B. Ward, 2018: Turbulence scaling comparisons in the ocean surface boundary layer. J. Geophys. Res. Oceans, 123, 2172-2191, https://doi.org/ 10.1002/2017JC013525.

Francois, M. M., S. J. Cummins, E. D. Dendy, D. B. Kothe, J. M. Sicilian, and M. W. Williams, 2006: A balanced-force algorithm for continuous and sharp interfacial surface tension models within a volume tracking framework. J. Comput Phys., 213, 141-173, https://doi.org/10.1016/j.jcp.2005.08.004.

Gemmrich, J. R., 2010: Strong turbulence in the wave crest region. J. Phys. Oceanogr., 40, 583-595, https://doi.org/10.1175/ 2009JPO4179.1.

— , and D. Farmer, 2004: Near-surface turbulence in the presence of breaking waves. J. Phys. Oceanogr., 34, 1067-1086, https:// doi.org/10.1175/1520-0485(2004)034<1067:NTITPO > 2.0.CO;2.

— T. Mudge, and V. Polonichko, 1994: On the energy input from wind to surface waves. J. Phys. Oceanogr., 24, 2413-2417, https:// doi.org/10.1175/1520-0485(1994)024<2413:OTEIFW>2.0.CO;2.

_, M. L. Banner, and C. Garrett, 2008: Spectrally resolved energy dissipation rate and momentum flux of breaking waves. J. Phys. Oceanogr., 38, 1296-1312, https://doi.org/10.1175/2007JPO3762.1.

Hwang, P. A., and M. A. Sletten, 2008: Energy dissipation of windgenerated waves and whitecap coverage. J. Geophys. Res Oceans, 113, C02012, https://doi.org/10.1029/2007JC004277.

Kirby, J. T., and M. Derakhti, 2019: Short-crested wave breaking. Eur. J. Mech. B Fluids, 73, 100-111, https://doi.org/10.1016/ j.euromechflu.2017.11.001.

Kleiss, J. M., and W. K. Melville, 2010: Observations of wave breaking kinematics in fetch-limited seas. J. Phys. Oceanogr., 40, 2575-2604, https://doi.org/10.1175/2010JPO4383.1.

Lamarre, E., and W. Melville, 1991: Air entrainment and dissipation in breaking waves. Nature, 351, 469-472, https://doi.org/ 10.1038/351469a0.

Ma, G., F. Shi, and J. T. Kirby, 2011: A polydisperse two-fluid model for surf zone bubble simulation. J. Geophys. Res., 116, C05010, https://doi.org/10.1029/2010JC006667. 
Melville, W. K., 1996: The role of surface-wave breaking in air-sea interaction. Annu. Rev. Fluid Mech., 28, 279-321, https:// doi.org/10.1146/annurev.fl.28.010196.001431.

— - and P. Matusov, 2002: Distribution of breaking waves at the ocean surface. Nature, 417, 58-63, https://doi.org/10.1038/ 417058a.

Mori, N., T. Suzuki, and S. Kakuno, 2007: Noise of acoustic doppler velocimeter data in bubbly flows. J. Eng. Mech., 133, 122-125, https://doi.org/10.1061/(ASCE)0733-9399(2007)133:1(122).

Phillips, O. M., 1985: Spectral and statistical properties of the equilibrium range in wind-generated gravity waves. J. Fluid Mech., 156, 505-531, https://doi.org/10.1017/S0022112085002221.

Pizzo, N., W. K. Melville, and L. Deike, 2019: Lagrangian transport by nonbreaking and breaking deep-water waves at the ocean surface. J. Phys. Oceanogr., 49, 983-992, https://doi.org/ 10.1175/JPO-D-18-0227.1.

Rapp, R. J., and W. K. Melville, 1990: Laboratory measurements of deep-water breaking waves. Philos. Trans. Roy. Soc. London, 331A, 735-800, https://doi.org/10.1098/rsta.1990.0098.

Romero, L., W. K. Melville, and J. M. Kleiss, 2012: Spectral energy dissipation due to surface wave breaking. J. Phys. Oceanogr., 42, 1421-1444, https://doi.org/10.1175/JPO-D-11-072.1.

Scanlon, B., and B. Ward, 2016: The influence of environmental parameters on active and maturing oceanic whitecaps. J. Geophys. Res. Oceans, 121, 3325-3336, https://doi.org/ 10.1002/2015JC011230.

Schwendeman, M., and J. Thomson, 2015: Observations of whitecap coverage and the relation to wind stress, wave slope, and turbulent dissipation. J. Geophys. Res. Oceans, 120, 83468363, https://doi.org/10.1002/2015JC011196.

,$- \ldots$, and J. Gemmrich, 2014: Wave breaking dissipation in a young wind sea. J. Phys. Oceanogr., 44, 104-127, https:// doi.org/10.1175/JPO-D-12-0237.1.

Sutherland, P., and W. K. Melville, 2013: Field measurements and scaling of ocean surface wave-breaking statistics. Geophys. Res. Lett., 40, 3074-3079, https://doi.org/10.1002/GRL.50584.

, and — 2015: Field measurements of surface and near-surface turbulence in the presence of breaking waves. J. Phys. Oceanogr., 45, 943-965, https://doi.org/10.1175/JPO-D-14-0133.1.
Terray, E., M. Donelan, Y. Agrawal, W. Drennan, K. Kahma, A. Williams, P. Hwang, and S. Kitaigorodskii, 1996: Estimates of kinetic energy dissipation under breaking waves. J. Phys. Oceanogr., 26, 792-807, https://doi.org/10.1175/1520-0485(1996) 026<0792:EOKEDU > 2.0.CO;2.

Thomson, J., 2012: Wave breaking dissipation observed with SWIFT drifters. J. Atmos. Oceanic Technol., 29, 1866-1882, https://doi.org/10.1175/JTECH-D-12-00018.1.

— , and A. Jessup, 2009: A Fourier-based method for the distribution of breaking crests from video observations. J. Atmos. Oceanic Technol., 26, 1663-1671, https://doi.org/10.1175/ 2009JTECHO622.1.

- M. S. Schwendeman, S. F. Zippel, S. Moghimi, J. Gemmrich, and W. E. Rogers, 2016: Wave-breaking turbulence in the ocean surface layer. J. Phys. Oceanogr., 46, 1857-1870, https:// doi.org/10.1175/JPO-D-15-0130.1.

Tian, Z., M. Perlin, and W. Choi, 2010: Energy dissipation in twodimensional unsteady plunging breakers and an eddy viscosity model. J. Fluid Mech., 655, 217-257, https://doi.org/10.1017/ S0022112010000832.

Wang, D. W., and H. W. Wijesekera, 2018: Observations of breaking waves and energy dissipation in modulated wave groups. J. Phys. Oceanogr., 48, 2937-2948, https://doi.org/ 10.1175/JPO-D-17-0224.1.

Wu, C. H., and H. M. Nepf, 2002: Breaking criteria and energy losses for three-dimensional wave breaking. J. Geophys. Res., 107, 3177, https://doi.org/10.1029/2001JC001077.

Zappa, C. J., W. R. McGillis, P. A. Raymond, J. B. Edson, E. J. Hintsa, H. J. Zemmelink, J. W. H. Dacey, and D. T. Ho, 2007: Environmental turbulent mixing controls on air-water gas exchange in marine and aquatic systems. Geophys. Res. Lett., 34, L10601, https://doi.org/10.1029/2006GL028790.

_, M. L. Banner, R. P. Morison, and S. E. Brumer, 2016: On the variation of the effective breaking strength in oceanic sea states. J. Phys. Oceanogr., 46, 2049-2061, https://doi.org/ 10.1175/JPO-D-15-0227.1.

Zippel, S. F., J. Thomson, and G. Farquharson, 2018: Turbulence from breaking surface waves at a river mouth. J. Phys. Oceanogr., 48, 435-453, https://doi.org/10.1175/JPO-D-17-0122.1. 